\title{
Gold nanorod-based poly(lactic-co-glycolic acid) with manganese dioxide core-shell structured multifunctional nanoplatform for cancer theranostic applications
}

\author{
This article was published in the following Dove Press journal: \\ International Journal of Nanomedicine \\ 13 April 2017 \\ Number of times this article has been viewed
}

\author{
Lei Wang ${ }^{1-3}$ \\ Dong $\mathrm{Li}^{1,2}$ \\ Yongwei $\mathrm{Hao}^{1,2}$ \\ Mengya $\mathrm{Niu}^{1,2}$ \\ Yujie $\mathrm{Hu}^{1,2}$ \\ Hongjuan Zhao',2 \\ Junbiao Chang ${ }^{2,3}$ \\ Zhenzhong Zhang ${ }^{1,2}$ \\ Yun Zhang ${ }^{1,2}$ \\ 'School of Pharmaceutical Sciences, \\ Zhengzhou University, ${ }^{2} \mathrm{Key}$ \\ Laboratory of Targeting Therapy and \\ Diagnosis for Critical Disease, Henan \\ Province, ${ }^{3}$ School of Chemistry and \\ Molecular Engineering, Zhengzhou \\ University, Zhengzhou, People's \\ Republic of China
}

Correspondence: Zhenzhong Zhang;

Yun Zhang

School of Pharmaceutical Sciences,

Zhengzhou University, 100 Kexue

Avenue, Zhengzhou, 45000I,

People's Republic of China

Tel +86 37। 67781910

Fax +86 37। 67781908

Email zhangzz0I@।26.com;

zhang_yun@ymail.com

\begin{abstract}
Recently, photothermal therapy has become a promising strategy in tumor treatment. However, the therapeutic effect was seriously hampered by the low tissue penetration of laser. Therefore, in this study, radiofrequency (RF) with better tissue penetration was used for tumor hyperthermia. First, one type of gold nanorods (AuNRs) suitable for RF hyperthermia was selected. Then, poly(lactic-co-glycolic acid) (PLGA) nanoparticles (NPs) loaded with AuNRs and docetaxel (DTX) (PLGA/AuNR/DTX) NPs were constructed. Finally, manganese dioxide $\left(\mathrm{MnO}_{2}\right)$ ultrathin nanofilms were coated on the surfaces of PLGA/AuNR/DTX NPs by the reduction of $\mathrm{KMnO}_{4}$ to construct the PLGA/AuNR/DTX@ $\mathrm{MnO}_{2}$ drug delivery system. This drug delivery system can not only be used for the combined therapy of chemotherapy and RF hyperthermia but can also produce $\mathrm{Mn}^{2+}$ to enable magnetic resonance imaging. Furthermore, the RF hyperthermia and the degradation of $\mathrm{MnO}_{2}$ can significantly promote the controlled drug release in a tumor region. The in vitro and in vivo results suggested that the PLGA/AuNR/ DTX@ $\mathrm{MnO}_{2}$ multifunctional drug delivery system is a promising nanoplatform for effective cancer theranostic applications.
\end{abstract}

Keywords: poly(lactic-co-glycolic acid), gold nanorod, manganese dioxide, radiofrequency, hyperthermia, dual-mode imaging, controlled release

\section{Introduction}

Cancer has a serious threat to the health and life of human beings. Currently, the main measures of treatment include chemotherapy, surgical treatment, and radiation therapy. ${ }^{1,2}$ However, there are many disadvantages in these therapies, which lead to the nonideal therapeutic effect. ${ }^{3}$ With the development of tumor adjunctive therapy, the combined therapy of hyperthermia and chemotherapy has become a hot spot in related research fields. ${ }^{4,5}$ Gold nanomaterials are a type of biomaterials that exhibit many excellent properties, such as easy molding, easy modification, and low toxicity, and they have always been used for near-infrared (NIR) light-induced photothermal therapy (PTT) ${ }^{6,7}$ However, low tissue penetration and skin burning of NIR laser have seriously hindered its use. ${ }^{8}$

Using radiofrequency (RF) is a handy and effective method to solve the abovementioned problems. Moreover, at present, RF hyperthermia has been used as an auxiliary clinical treatment for cancer. ${ }^{9,10}$ It brings about several advantages for cancer therapy, including simple operation, no wound, and full-body penetration. 
However, long-time RF hyperthermia is also harmful to a person's health due to the electromagnetic wave emitted by an RF. Shortening the treatment time, reducing the treatment damage, and improving the treatment effect are urgently needed. Therefore, finding an effective sensitizer of RF hyperthermia is the key. Gold nanorods (AuNRs) are rod-like gold nanomaterials that are always used for lightinduced photothermal (photodynamic) therapy. ${ }^{11}$ Song et al used AuNRs for doxorubicin loading and PTT. ${ }^{12}$ Wang et al reported that the modified AuNRs had unique optical and photothermal properties, making them promising nanostructures for biomedical applications. ${ }^{13}$ Recently, Collins et al reported that gold nanoparticles (NPs) can be used for RF-induced hyperthermia. ${ }^{14}$ Rejinold et al summarized many nanomaterials (including gold nanomaterials), which can be used for RF hyperthermia in vitro. ${ }^{15}$ However, the present study found that AuNRs with different aspect ratios (ARs) have different heating efficiencies induced by RF; therefore, in this study, AuNRs with a suitable AR were selected for RF-induced hyperthermia in vitro and in vivo.

It was reported that cetyltrimethylammonium bromide (CTAB) used for the construction of AuNRs always causes obvious toxicity to the normal cells. ${ }^{16}$ Moreover, as a commonly used anti-tumor chemotherapeutic drug in the clinical application, docetaxel (DTX) is restricted due to its high hydrophobicity and severe side effects. ${ }^{17,18}$ Therefore, a suitable delivery system should be prepared to reduce the toxicity of CTAB and DTX to the normal cells and tissues. Poly(lactic-co-glycolic acid) (PLGA) NPs are a family of US Food and Drug Administration-approved biodegradable polymers that are physically strong and highly biocompatible and have been extensively studied as delivery vehicles for drugs, proteins, and various other macromolecules. ${ }^{19}$ Thus, PLGA NPs were selected for loading AuNRs and DTX, and should be able to provide chemotherapy and RF hyperthermia at the same time.

Compared with the normal tissues, tumor microenvironment has unique characteristics, such as lower $\mathrm{pH}$ value and higher glutathione (GSH) level..$^{20,21}$ In a previous study, a type of multifunctional nanosheet based on manganese dioxide $\left(\mathrm{MnO}_{2}\right)$ was prepared, and it was shown that $\mathrm{MnO}_{2}$ nanosheets could respond to a slightly acidic environment and high concentration of reduced GSH, which caused the degradation of $\mathrm{MnO}_{2}$ into manganese ions enabling $T_{1}$-weighted magnetic resonance imaging (MRI). ${ }^{22}$ Decorating $\mathrm{MnO}_{2}$ sheets on the surface of PLGA could bring the MRI function and controlled release function to the nanoplatform in the tumor sites. There are some studies on the combination therapy of GSH-sensitive controlled release and PTT. Han et al synthesized a novel conjugate by conjugating paclitaxel to paclitaxel by using disulfide linkage. The conjugate could self-assemble into uniform NPs with 1,1-dioctadecyl-3,3,3,3tetramethylindotricarbocyanine iodide encapsulated for PTT, which showed high antitumor effect. ${ }^{23}$ Hou et al used $-\mathrm{S}-\mathrm{s}-$ to construct a GSH-sensitive controlled release drug delivery system for the combined PTT, by using the carbon nanotubes. ${ }^{24}$ It was reported that the $-\mathrm{s}-\mathrm{s}-$ bond used in many nano drug delivery systems was always used for redox-responsive releasing, and some of these systems can be used for combined PTT, indicating that the combined therapy of redox-responsive releasing and hyperthermia is an effective method for tumor inhibition. ${ }^{25,26}$ The aim of the present study was to prepare a new kind of core-shell drug delivery system (PLGA/AuNR/DTX@ $\mathrm{MnO}_{2}$ ). As illustrated in Figure 1, the AuNRs with a suitable AR and DTX were loaded in PLGA NPs by using the emulsion solvent evaporation method. Then, $\mathrm{MnO}_{2}$ ultrathin nanosheets were loaded on the surface of PLGA to obtain PLGA/AuNR/DTX@ $@ \mathrm{MnO}_{2}$ drug delivery system. The efficiency of the multifunctional nanoplatform was assessed in vitro and in vivo, and the results demonstrated the combined therapeutic effect of RF hyperthermia and chemotherapy, the controlled release effect, and an MRI effect in cancer theranostic applications.

\section{Materials and methods Materials}

PLGA (lactide $/$ glycolide $=50: 50$, molecular weight $=94,000$ Da) was purchased from Daigang Biomaterial Co., Ltd. (Jinan, People's Republic of China). Chloroauric acid $\left(\mathrm{HAuCl}_{4}\right)$ was obtained from Sinopharm Chemical Reagent Co., Ltd. (Shanghai, People's Republic of China). CTAB was obtained from Dibo Ltd. (Shanghai, People's Republic of China). Ascorbic acid (AA), sodium borohydride $\left(\mathrm{NaBH}_{4}\right)$, and silver nitrate $\left(\mathrm{AgNO}_{3}\right)$ were purchased from Guobang Pharmaceutical Company (Shanxi, People's Republic of China). GSH (purity >98\%) was obtained from Genview Co., Ltd. (Beijing, People's Republic of China). DTX was obtained from Meilun Biotechnological Company (Dalian, People's Republic of China). Poly(vinyl alcohol) (PVA) was obtained from Kelong Chemical Reagent Company (Chengdu, People's Republic of China). Both potassium permanganate $\left(\mathrm{KMnO}_{4}\right)$ and 2-(N-morpholino) ethanesulfonic acid (MES) were purchased from Sinopharm Chemical Reagent Co., Ltd. (Shanghai, People's Republic of China). The Michigan Cancer Foundation - 7 (MCF-7) human breast cancer cell line was purchased commercially 


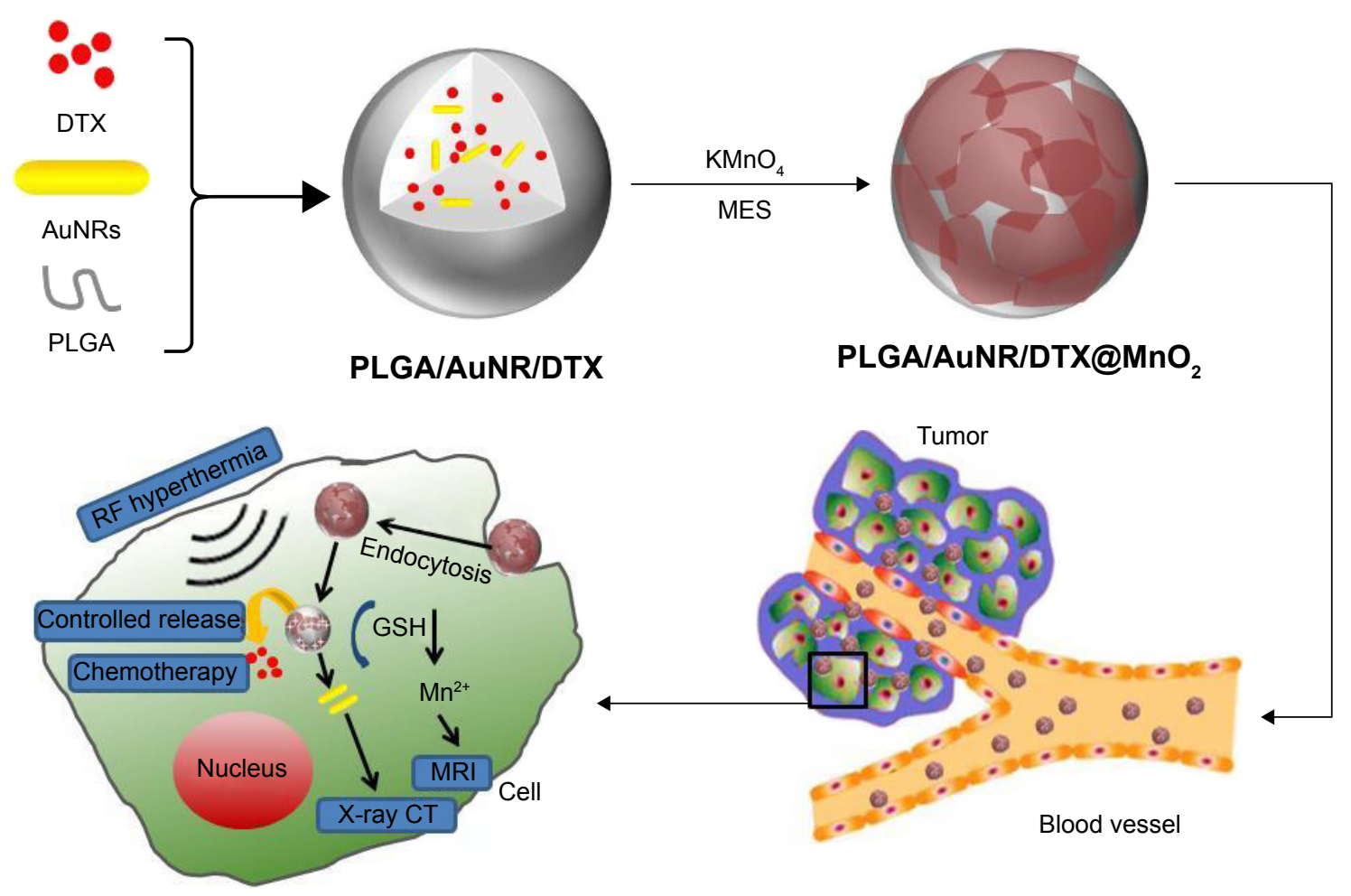

Figure I Scheme of PLGA/AuNR/DTX@MnO and its biofunctions.

Abbreviations: AuNR, gold nanorod; CT, computed tomography; DTX, docetaxel; GSH, glutathione; KMnO, potassium permanganate; MES, 2-(N-morpholino) ethanesulfonic acid; MRI, magnetic resonance imaging; $\mathrm{MnO}_{2}$, manganese dioxide; PLGA, poly(lactic-co-glycolic acid); RF, radiofrequency.

from the Cell Bank of the Chinese Academy of Sciences (Catalog No TCHu 74; Shanghai, People's Republic of China). All the other chemicals were obtained from SigmaAldrich Co. Ltd (St Louis, MO, USA).

\section{Preparation of the gold seeds solution}

First, $0.5 \mathrm{~mL}$ of $\mathrm{HAuCl}_{4}(0.5 \mathrm{mM})$ was added drop by drop to $5 \mathrm{~mL}$ of CTAB solution $(0.2 \mathrm{M})$ under magnetic stirring with a rate of 550 cycles/min at an ambient temperature. Second, chilled $\mathrm{NaBH}_{4}(0.6 \mathrm{~mL}, 0.01 \mathrm{M})$ was added to the abovementioned mixture solution. When the color of the mixing solution changed from pale yellow to claybank, the seed solution was stirred vigorously for 2 min and kept at room temperature for $30 \mathrm{~min}$ away from light for further use.

\section{Preparation of the growth solution}

First, $8.0 \mathrm{~mL}$ of $\mathrm{HAuCl}_{4}(0.5 \mathrm{mM})$ was mixed with $5.0 \mathrm{~mL}$ CTAB $(0.20 \mathrm{M})$ under slight stirring, and then, different volumes of $0.004 \mathrm{M} \mathrm{AgNO}_{3}$ solution (20, 40, 100, 280, and $360 \mu \mathrm{L}$ ) were added to the mixture at room temperature. Then, $140 \mu \mathrm{L}$ AA of $0.0788 \mathrm{M}$ was added drop by drop into the five samples. Following the addition of AA, the colors of the growth solutions changed from dark yellow, and the solutions became colorless.

\section{Preparation of AuNRs}

According to the CTAB-induced seed-mediated growth method, ${ }^{27,28}$ the AuNRs (AR =1.48:1, 2.34:1, 3.14:1, 3.52:1, and 4.20:1) were synthesized.

First, $1 \mathrm{~mL}$ of gold seed solution was added into the growth solution and stirred at 350 cycles $/ \mathrm{min}$. The colors of the solutions gradually changed within $\sim 10-20 \mathrm{~min}$. The mixture was kept for $1 \mathrm{~h}$ at room temperature, and the AuNRs products were obtained after centrifugation $(12,000 \mathrm{rpm} ; 15 \mathrm{~min})$ and rinsing three times to remove the excess CTAB.

In order to select the most suitable AR of AuNRs for RF hyperthermia, the Au concentration of each AuNRs sample was set at $100 \mu \mathrm{g} / \mathrm{mL}$. Subsequently, the samples were exposed to an RF field (13.56 MHz; $400 \mathrm{~W})$ for $5 \mathrm{~min}$, and the temperatures were recorded. Finally, the AuNRs sample with the best heat production was chosen for further experiments.

\section{Construction of PLGA/AuNR/DTX}

The PLGA/AuNR/DTX NPs were constructed by using the emulsion solvent evaporation method reported in the previous studies. ${ }^{29,30}$ In order to prepare the organic phase, PLGA (100 mg) and DTX (6 mg) were dissolved into acetone 
( $5 \mathrm{~mL})$. Furthermore, $5 \mathrm{~mL}$ of AuNRs $(2 \mathrm{mg} / \mathrm{mL})$ were dissolved in $10 \mathrm{mg} / \mathrm{mL}$ PVA $(20 \mathrm{~mL})$ to obtain the aqueous phase. Then, the organic phase was added drop by drop into the aqueous phase in a magnetic stirrer with a rate of $550 \mathrm{rpm}$ at room temperature for $\sim 4 \mathrm{~h}$ for complete acetone evaporation. Finally, PLGA/AuNR/DTX NPs were collected by centrifugation $(15,000 \mathrm{~g})$ for $30 \mathrm{~min}$ and washed four times with deionized water.

\section{Preparation of PLGA/AuNR/DTX@ $\mathrm{MnO}_{2}$}

$\mathrm{MnO}_{2}$ nanosheets were loaded on the surface of the PLGA/ AuNR/DTX NPs by using the MES oxidation-reduction method. ${ }^{31}$ Briefly, the above PLGA/AuNR/DTX NPs were added into $6.25 \mathrm{~mL}$ MES buffer $\left(100 \times 10^{-3} \mathrm{M}\right.$; $\left.\mathrm{pH}=6.0\right)$ under ultrasonic conditions, and then, $\mathrm{KMnO}_{4}$ solution ( $5 \mathrm{mM}$ ) was added and mixed for $\sim 5 \mathrm{~min}$. The above mixture was subjected to dialysis for $12 \mathrm{~h}$ to remove the byproducts. After centrifugation, PLGA/AuNR/DTX@, $\mathrm{MnO}_{2}$ NPs were collected and concentrated by freeze-drying for further use.

\section{Characterization}

Small drops of AuNRs with different ARs, PLGA/DTX, PLGA/AuNR/DTX, and PLGA/AuNR/DTX@MnO, solutions were placed on the grids. Transmission electron microscopy (TEM; JEM-1200 EX, 120 kV) and cryogenic TEM (JEOL $2100 \mathrm{HC}$; Tokyo, Japan) were used to observe the morphologies of the solutions. The energy-dispersive X-ray (EDX) spectroscopy was also tested to analyze the chemical elements of the NPs. The optical properties of NPs were characterized by using an ultraviolet-visible (UV-Vis) spectrometer (UV-Vis 2700; Shimadzu Co. Ltd., Kyoto, Japan). The temperatures of AuNRs (Au concentration $=100 \mu \mathrm{g} / \mathrm{mL}$ ) exposed to RF were monitored by using an Infrared Thermometer (IR 97; HuaShengChang Instrument Co. Ltd.; Shenzhen, People's Republic of China). X-ray photoelectron spectroscopy (XPS) was used to analyze the chemical properties of Mn. XPS data were processed with XPSPEAK software, Version 4.1. The size distribution and zeta potential of the NPs with or without $10 \%$ fetal bovine serum were measured by using dynamic light scattering (DLS; ZS-90; Malvern Instruments, Worcestershire, UK). DLS was also used to determine the stability of PLGA/AuNR/DTX@ $\mathrm{MnO}_{2}$ NPs in $10 \%$ fetal bovine serum.

The PLGA/AuNR/DTX@ $\mathrm{MnO}_{2}$ NPs were dissolved in phosphate-buffered saline (PBS; $\mathrm{pH}=7.4$ ) containing $0 \mathrm{mM}$ and $2 \mathrm{mM}$ GSH. Then, $2 \mathrm{~mL}$ of this solution was separately dropped into a 24-well cell culture plate. After $4 \mathrm{~h}$, in vitro
$T_{1}$-weighted MR images were obtained with a 1.5 -T clinical MR scanner (Signa Excite; GE Healthcare Systems, Milwaukee, WI, USA). A MicroMR instrument (0.5 T; Shanghai Niumag Electronic Technology Co., Ltd., Shanghai, People's Republic of China) was used for the analysis of the longitudinal $\left(T_{1}\right)$ relaxivity values.

\section{Determination of loading and release rates}

The $\mathrm{MnO}_{2}$ shells of PLGA/AuNR/DTX@ $\mathrm{MnO}_{2}$ NPs were first digested by adding dithiothreitol. The encapsulation ratio of DTX was determined according to the method reported by a prior research. ${ }^{32}$ First, DTX was extracted from PLGA/AuNR/DTX NPs by adding the digested NPs into the mixture of methanol and acetonitrile $(\mathrm{v} / \mathrm{v}=1: 3)$. After sonication for $30 \mathrm{~min}$, the suspension was centrifuged, and the collected supernatant was measured by using high performance liquid chromatograph (HPLC, Agilent 1200, Agilent Technologies Inc., Santa Clara, CA, USA) in order to detect the concentration of DTX. A reverse-phase Intertex C18 column (Sciencehome, $5 \mu \mathrm{m}, 4.6 \times 250 \mathrm{~mm}$ ) was used as an analytical column, and the flow rate of mobile phase was set at $1 \mathrm{~mL} / \mathrm{min}$. The mobile phase consisting of deionized water and methanol $(\mathrm{v} / \mathrm{v}=27: 73)$ was applied. DTX eluting from the column was detected by using a UV detector set at $231 \mathrm{~nm}$. The encapsulation ratio was calculated. Besides this, the $\mathrm{MnO}_{2}$ and AuNR contents were measured by using inductively coupled plasma-atomic emission spectrometry (ICP-AES).

For the in vitro release study, PLGA/AuNR/DTX and PLGA/AuNR/DTX@MnO ${ }_{2}$ samples were placed separately into dialysis bags (cutoff mass $=12,000 \mathrm{Da}$ ), which were dialyzed in $35 \mathrm{~mL}$ PBS $(0.1 \mathrm{M}$; $\mathrm{pH}=7.4$ or 5.0$)$ containing $0.5 \%$ sodium dodecyl sulfonate at $37^{\circ} \mathrm{C}$ with a stirring rate of $100 \mathrm{rpm}$. Both the samples were divided into two groups: experimental group and control group. The PBS in the control group contained $2 \mathrm{mM} \mathrm{GSH}$, and the other did not contain GSH. During the experimental process, $0.5 \mathrm{~mL}$ of solution was drawn from the solution outside the dialysis bags at 15 and $30 \mathrm{~min}$ and 1, 2, 4, 6, 8, 12, 24, 36, 48, and $72 \mathrm{~h}$. Meanwhile, the same volume of the release medium solution was replenished. The concentration of DTX released from NPs into release medium was quantified by using an HPLC under the above chromatographic conditions.

\section{Cellular experiments}

\section{Cell culture}

The MCF-7 human breast cancer cell line was cultured in Dulbecco's Modified Eagle's Medium with 1\% 
penicillin/streptomycin and $10 \%$ fetal bovine serum at $37^{\circ} \mathrm{C}$ in a humidified incubator.

\section{Cellular uptake}

In order to determine the cellular uptake ratio of PLGA/ AuNR/DTX@ $\mathrm{MnO}_{2}$, fluorescein isothiocyanate (FITC) was used for fluorescence tracing. The FITC-labeled NPs were prepared by using the abovementioned steps (in "Construction of PLGA/AuNR/DTX" and "Preparation of PLGA/ AuNR/DTX@ $\mathrm{MnO}_{2}$ " sections). FITC was added in the organic phase in each group with the final concentration of $0.125 \mu \mathrm{g} / \mathrm{mL}$. The cells were cultured on the 6-well plates for $24 \mathrm{~h}$ and then exposed to FITC, PLGA/AuNR/DTX, and PLGA/AuNR/DTX@MnO ${ }_{2} \mathrm{NP}$ solution. After incubation for $5 \mathrm{~h}$, the old medium of different samples was removed and the cells were washed with PBS for twice. Finally, the cells were counterstained with 4',6-diamidino-2-phenylindole (DAPI; $1.2 \mu \mathrm{g} / \mathrm{mL}$ ) for $20 \mathrm{~min}$ and then imaged with a laser confocal microscope (Fluoview V1000, Olympus Optical Co., Ltd., Tokyo, Japan). For further evaluating the cellular uptake of the NPs, the above samples were analysed by using flow cytometry (FCM; Epics XL MCL ${ }^{\mathrm{TM}}$ ) three times.

\section{Cell viability}

MCF-7 cells with a density of $\sim 8.0 \times 10^{3}$ per well were inoculated in 96-well plates and cultivated for $24 \mathrm{~h}$. Subsequently, the culture medium was replaced with different concentrations of formulations diluted in fresh culture medium (DTX concentration $=0.5,1$, and $2 \mu \mathrm{g} / \mathrm{mL}$; Au concentration $=10,20$, and $40 \mu \mathrm{g} / \mathrm{mL}$ ). For the RF groups, the cells were exposed to a 13.56-MHz RF field for $5 \mathrm{~min}$, and then they were incubated with the preparations for $5 \mathrm{~h}$. Cell inhibition was measured by using sulforhodamine B assay after a further $24 \mathrm{~h}$ of incubation. ${ }^{33}$ The cells were divided into the following groups: DTX, PLGA/AuNR/MnO 2 , PLGA/DTX@ $\mathrm{MnO}_{2}$, and PLGA/ AuNR/DTX@ $\mathrm{MnO}_{2}$ groups with or without RF treatment.

\section{Cell apoptosis and GSH level}

MCF-7 cells with a density of $\sim 8.0 \times 10^{3}$ per well were inoculated in 96-well plates and cultivated for $24 \mathrm{~h}$. Then, the cells were incubated with DTX, PLGA/DTX, PLGA/AuNR, PLGA/AuNR/DTX, and PLGA/AuNR/DTX@ $\mathrm{MnO}_{2}(\mathrm{DTX}$ concentration $=2 \mu \mathrm{g} / \mathrm{mL}$; Au concentration $=40 \mu \mathrm{g} / \mathrm{mL}$ ) with or without RF treatment for $5 \mathrm{~h}$ and washed with PBS, and then, fresh medium was added. The treatment of RF groups was the same as described in the "Cell viability" section. The cells were cultured for another $24 \mathrm{~h}$, and then they were resuspended in $500 \mu \mathrm{L}$ of Annexin V-FITC binding buffer, stained with $5 \mu \mathrm{L}$ Annexin V-FITC and $5 \mu \mathrm{L}$ propidium iodide (PI) for $15 \mathrm{~min}$ in a dark environment, and finally analyzed by using a flow cytometer (Accuri C6, BD Co. Ltd., San Jose, CA, USA). In addition, the GSH change after the treatment of PLGA/AuNR/DTX@ $@ \mathrm{MnO}_{2}$ was detected by using the reduced GSH assay kit (Nanjing Jiancheng Bioengineering Institute, Nanjing, People's Republic of China).

\section{Animal experiments}

The animal studies were conducted on female Kunming mice ( 18-20 g), which were purchased from Henan Laboratory Animal Center. All the mice received care in compliance with the criteria of the National Regulation on the Management of Laboratory Animals. A subcutaneous S-180 tumor model was established, and when the tumor volume reached $\sim 80-100 \mathrm{~mm}^{3}$ ( $\sim 7$ days after tumor inoculation), the treatment was started. This study was approved by the Ethics Committee of Zhengzhou University.

\section{Tumor growth inhibition}

The mice were divided into the following groups (five mice per group): saline, DTX, PLGA/DTX, PLGA/AuNR, PLGA/AuNR/DTX, and PLGA/AuNR/DTX@ $\mathrm{MnO}_{2}$ (DTX dosage $=15 \mathrm{mg} / \mathrm{kg}$ ) with or without $\mathrm{RF}$ irradiation treatment. The preparations were intravenously injected into the tumor-bearing mice every other day. In addition, the body weights and the tumor sizes (the tumor volume $=0.5 \times$ [tumor length] $\times$ [tumor width $]^{2}$ ) were measured every other day. The RF treatment was performed by exposing the tumor site to the RF field (RF condition: $13.56 \mathrm{MHz} ; 400 \mathrm{~W} ; 5 \mathrm{~min}$ ) at $4 \mathrm{~h}$ after injection. After treatment for five times, the mice were sacrificed to collect the tumors for hematoxylin and eosin (H\&E) staining. Simultaneously, the tumors of each group were photographed and measured for cell apoptosis by using the terminal deoxynucleotidyl transferase dUTP nick end labeling (TUNEL) method. The morphological changes were observed under a fluorescence microscope (Eclipse 50; Nikon Co. Ltd., Tokyo, Japan).

\section{Biodistribution of PLGA/AuNR/DTX@MnO} The tumor-bearing mice were given DTX or PLGA/AuNR/ DTX@ $\mathrm{MnO}_{2}($ DTX dose $=15 \mathrm{mg} / \mathrm{kg})$, via a tail vein injection. After treatment for 1, 2, 4, 6, and $8 \mathrm{~h}$, the tissues/organs were collected and homogenized in buffer (acetonitrile to saline ratio $=1: 1$ ). DTX in tissues/organs was determined by using an HPLC (Agilent 1100, USA) under the following conditions: an Eclipse XDB-C18 column $(150 \times 4.6 \times 5.0 \mathrm{~mm})$, mobile-phase acetonitrile $/$ water $=50: 50$, column temperature $=30^{\circ} \mathrm{C}$, detection wavelength $=231 \mathrm{~nm}$, flow rate $=1.0 \mathrm{~mL} / \mathrm{min}$, and injection volume $=20 \mathrm{~mL}$. 


\section{MRI and X-ray imaging}

In order to determine the diagnostic potential of PLGA/ AuNR/DTX@MnO,nanoplatform, MRI and X-ray imaging properties were detected. For in vivo imaging, PLGA/AuNR/ DTX@MnO NPs $(200 \mu \mathrm{L}$; DTX concentration=15 mg/kg) were intravenously injected into the tumor-bearing mice. After injection of PLGA/AuNR/DTX@MnO, MRI and $\mathrm{X}$-ray imaging were conducted on a clinical 3-T MRI scanner and X-ray scanner, respectively.

\section{Statistical analysis}

Quantitative data were expressed as mean \pm standard deviation and analyzed by using the analysis of variance followed by Dunnett's posttest. $P$-values $<0.05$ were considered statistically significant.

\section{Results and discussion Characterization of AuNRs}

In recent years, due to excellent physical and chemical properties, AuNRs have attracted great attention. ${ }^{34}$ AuNRs with different ARs have different absorption wavelengths that can be used for a variety of purposes, such as PTT. ${ }^{35,36}$ However, because of some drawbacks of NIR PTT, the RF irradiation has been used in this study for AuNRs-based hyperthermia.
The TEM images (Figure 2A) indicate that AuNRs with different ARs $(1.5: 1,2.3: 1,3.1: 1,3.5: 1$, and 4.2:1) were synthesized successfully. Meanwhile, UV-Vis spectroscopic analysis proved that their localized surface plasmon resonance (LSPR) peak extends from $\sim 520$ to $\sim 840 \mathrm{~nm}$ in the NIR region (Figure $2 \mathrm{~B}$ ). It was reported that the electromagnetic wave can easily penetrate the sharp tip structure and participate in heat production. ${ }^{37}$ Furthermore, a previous study showed that, as a kind of electromagnetic wave, RF can induce LSPR for heat production by using gold nanostars. ${ }^{38}$ As shown in Figure 2C, the temperatures increased with an increase in the ARs of AuNRs, demonstrating that AuNRs can be used as an RF-induced thermal-sensitive agent, and the AuNRs with the AR of 4.2:1 were selected for further research.

\section{Characterization of PLGA/AuNR/ DTX@MnO,}

PLGA/AuNR/DTX@ $@ \mathrm{MnO}_{2}$ enclosing DTX and AuNRs were prepared by the emulsion solvent evaporation method, followed by coating with $\mathrm{MnO}_{2}$ nanosheets and through the reduction reaction (Figure 1). PVA was used in the process of construction of PLGA/AuNR/DTX NPs, which can maintain the stability of NPs. Furthermore, the hydroxyl groups of PVA could be used for the construction
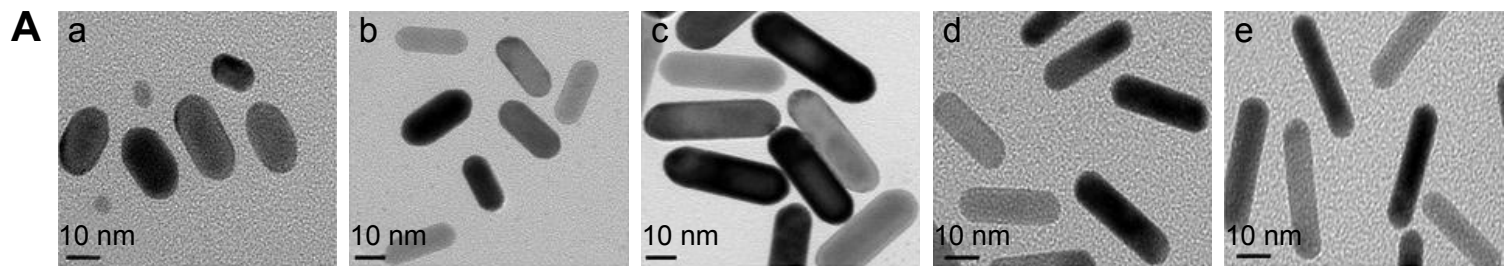

B

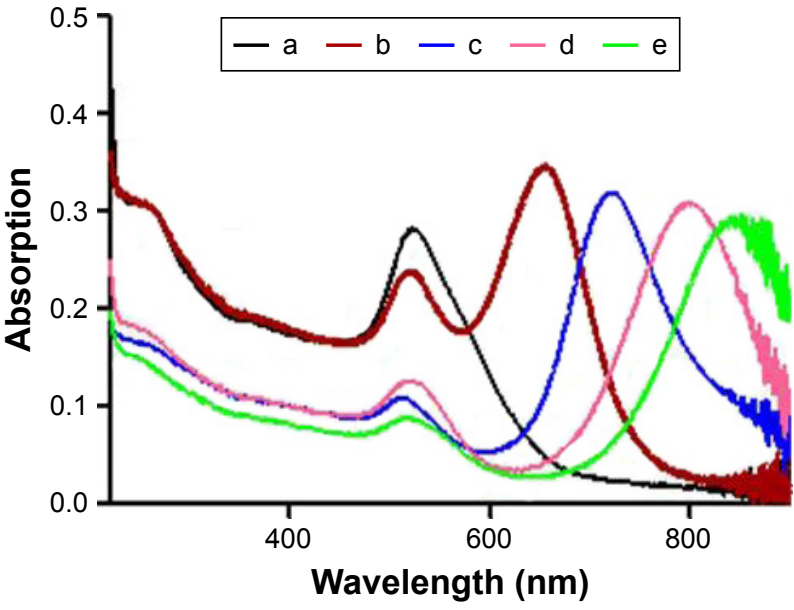

C

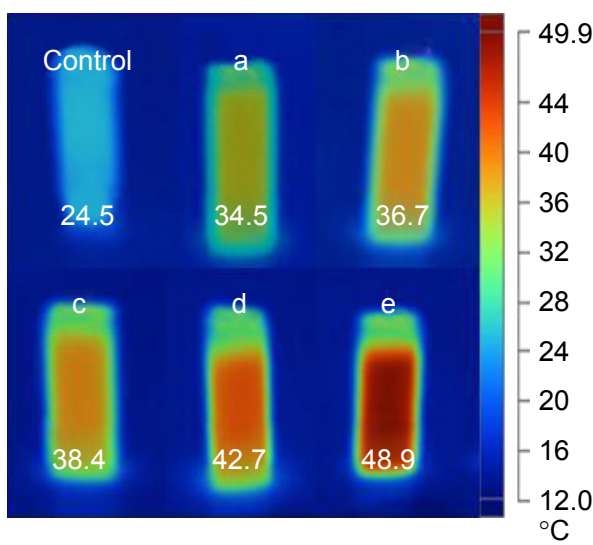

Figure 2 Characterization of AuNRs.

Notes: (A) TEM images of AuNRs with different ARs; (B) UV-Vis spectra of AuNRs with different ARs; (C) the temperatures (after RF irradiation) of AuNRs with different ARs. (a) 1.5:1; (b) 2.3:1; (c) 3.1:1; (d) 3.5:1; (e) 4.2:I.

Abbreviations: ARs, aspect ratios; AuNRs, gold nanorods; RF, radiofrequency; TEM, transmission electron microscopy; UV-Vis, ultraviolet-visible. 
of $\mathrm{MnO}_{2}$ on the PLGA/AuNR/DTX NPs through the reduction of $\mathrm{KMnO}_{4}$ by MES. ${ }^{39}$ The morphologies of PLGA/ DTX, PLGA/AuNR/DTX, and PLGA/AuNR/DTX@ $\mathrm{MnO}_{2}$ were determined by using TEM (Figure 3A). The results showed that all the three NPs showed clearly a spherical shape, and the AuNRs were wrapped in the PLGA NPs (Figure 3A-b). Then, the core-shell structure was constructed. The PLGA/AuNR/DTX NPs acted as the inner core, and the $\mathrm{MnO}_{2}$ nanosheets acted as the shell. It can be seen that after $\mathrm{MnO}_{2}$ nanosheets were coated on the surface of the PLGA/AuNR/DTX, the smooth surface of the NPs had become rough. In the whole process, the above three samples showed different colors (Figure 3B-a, b, and c).
The colors of PLGA/DTX, PLGA/AuNR/DTX, and PLGA/AuNR/DTX@MnO, were milky white, pink, and brown, respectively. Their average sizes were 239.0 \pm 3.7 , 263.3 \pm 4.5 , and $282.1 \pm 6.2 \mathrm{~nm}$, respectively (Figure 3B-d, e and $\mathrm{f}$ ), and the polymer dispersion index (PDI) values of them were $0.16 \pm 0.01,0.16 \pm 0.02$, and $0.18 \pm 0.02$, respectively. The zeta potential of the AuNRs was $27.4 \pm 2.2 \mathrm{mV}$, and this positive potential was attributed to CTAB that was coated on the surface of the AuNRs. ${ }^{40}$ However, the biotoxicity of CTAB and the low drug-loading rate of AuNRs are the obstacles for their use in the biomedical area. The PLGA NPs used in this study were not only used to decrease the toxicity of the AuNRs but also to increase the drug-loading rate. When the AuNRs and DTX were
A
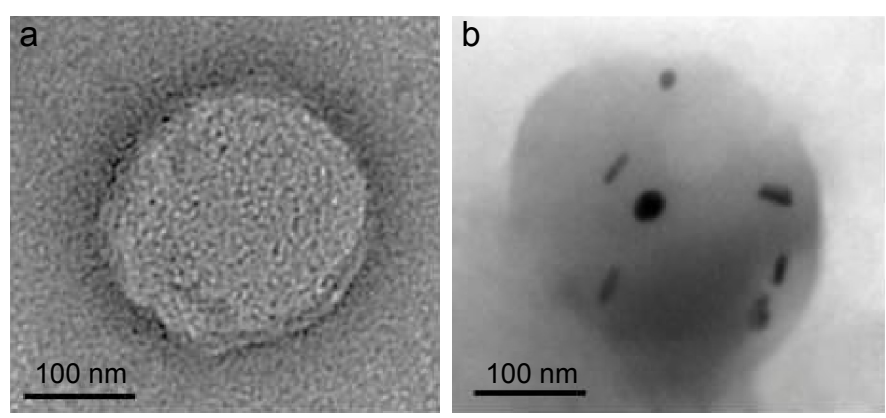

B
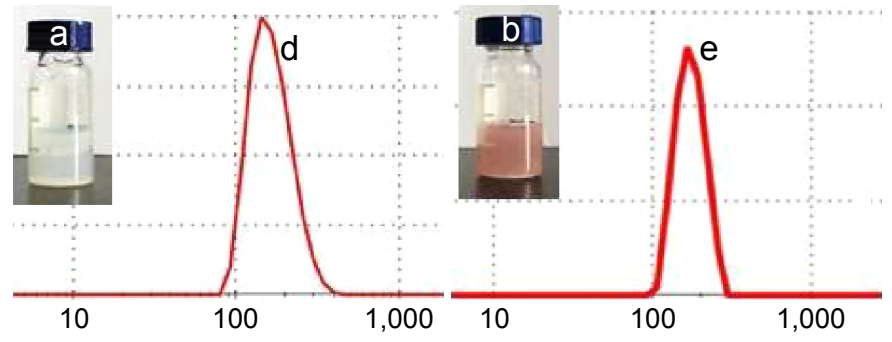

C

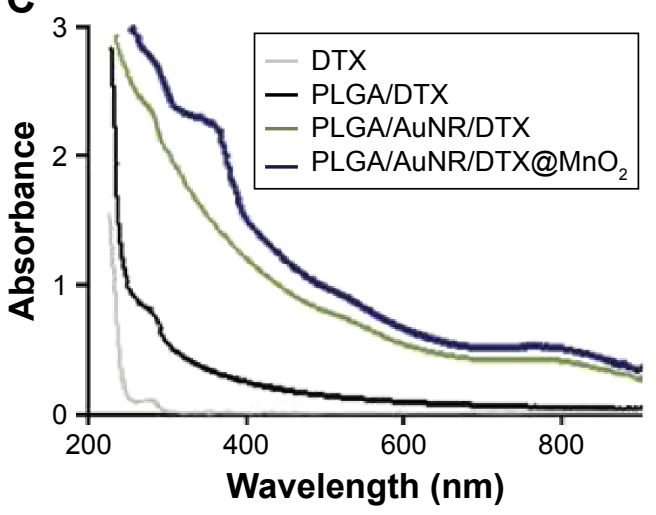

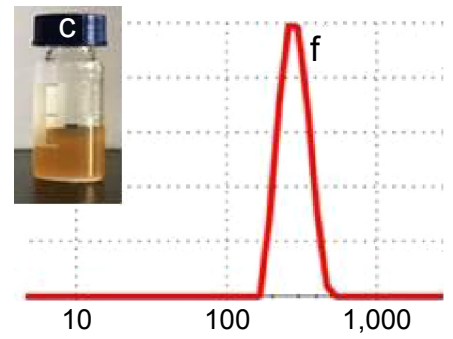

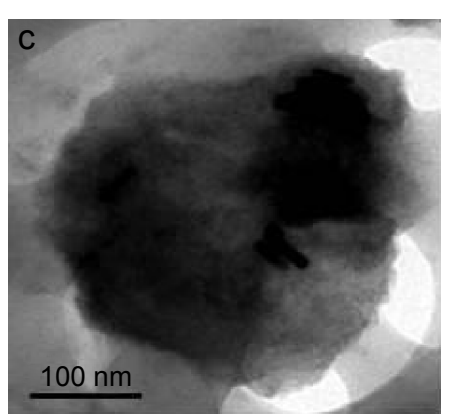

D

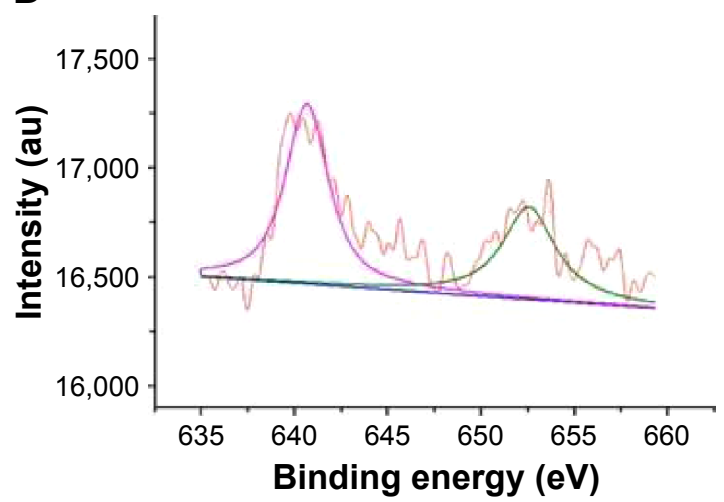

Figure 3 Characterization of PLGA/AuNRs/DTX@MnO.

Notes: (A) TEM images of the NPs: (a) PLGA/DTX, (b) PLGA/AuNR/DTX, and (c) PLGA/AuNR/DTX@MnO; (B) Photographs of the NPs: (a) PLGA/DTX, (b) PLGA/ AuNR/DTX, and (c) PLGA/AuNR/DTX@MnO ; the particle size of the NPs; (d) PLGA/DTX, (e) PLGA/AuNR/DTX, and (f) PLGA/AuNR/DTX@MnO ; (C) UV-Vis spectra of DTX, PLGA/DTX, PLGA/AuNR/DTX, and PLGA/AuNR/DTX@MnO; ; (D) XPS curve of the PLGA/AuNR/DTX@MnO NPs.

Abbreviations: AuNR, gold nanorod; DTX, docetaxel; $\mathrm{MnO}_{2}$, manganese dioxide; NPs, nanoparticles; PLGA, poly(lactic-co-glycolic acid); TEM, transmission electron microscopy; UV-Vis, ultraviolet-visible; XPS, X-ray photoelectron spectroscopy. 
loaded in PLGA NPs, the zeta potential of PLGA/AuNR/ DTX NPs was decreased to $-12.3 \pm 1.5 \mathrm{mV}$, and after loading the $\mathrm{MnO}_{2}$ nanosheets, the zeta potential changed to $-9.7 \pm 1.4 \mathrm{mV}$. Figure $\mathrm{S} 1$ shows the low magnification view of PLGA/AuNR/DTX@ $\mathrm{MnO}_{2}$, indicating that these NPs were nonaggregated. Furthermore, the stability of PLGA/AuNR/DTX@MnO ${ }_{2}$ NPs in a culture medium containing 10\% fetal bovine serum was also determined, for $24 \mathrm{~h}$. Tables S1 and S2 indicate that the particle size and zeta potential were broadly unchanged in the physiologically mimicked environment.

The construction of $\mathrm{MnO}_{2}$ shell was proved by the UVVis spectrum and XPS detection. As in Figure 3C, after the AuNRs were loaded in the PLGA/DTX NPs, the new peak $\sim 800 \mathrm{~nm}$ was obtained, which was attributed to the AuNRs. Compared with the PLGA/AuNR/DTX NPs, the $\mathrm{MnO}_{2}$ nanosheets showed a new wide peak between 300 and $400 \mathrm{~nm}$. For further determination, the XPS results (Figure 3D) indicate that the Mn 2p 3/2 and 2 p 1/2 were found to be 640.7 and $652.5 \mathrm{eV}$, respectively. These results were consistent with the reported data for $\mathrm{MnO}_{2} \cdot{ }^{32}$ After the $\mathrm{MnO}_{2}$ nanosheets were constructed, the EDX spectrum (Figure 4A) of the PLGA/AuNR/DTX@ $\mathrm{MnO}_{2}$ showed that the signal corresponding to $\mathrm{Mn}$ appeared. This result combined with all the above results, shown in Figure 3, indicated that the PLGA/AuNR/DTX@MnO ${ }_{2}$ delivery system was constructed successfully. The encapsulation ratio of DTX can reach $74.4 \%$, which may be due to its hydrophobic property. The results of ICP-AES determination indicated that the AuNRs and $\mathrm{MnO}_{2}$ content in the NPs were $\sim 8.2 \%$ and $5.0 \%$ in weight.

\section{Determination of the drug release}

In order to investigate the GSH and $\mathrm{pH}$-triggered drug release, PLGA/AuNR/DTX NPs and PLGA/AuNR/DTX@ $@ \mathrm{MnO}_{2}$ NPs were detected in the releasing medium with or without 2 $\mathrm{mM}$ GSH for the imitation of the physiological environment at a $\mathrm{pH}$ of 7.4 or 5.0 in vitro (Figure 4B). It was found that PLGA/AuNR/DTX NPs showed a similar release property with or without GSH treatment, and they also showed similar release property at a $\mathrm{pH}$ of 7.4 or 5.0 environment $(P>0.05)$. As shown in Figure 4B, the PLGA/AuNR/DTX NPs rapidly released the encapsulated DTX within $12 \mathrm{~h}$, and the cumulative release rate was $\sim 82.1 \%$. However, the release rate of PLGA/AuNR/DTX@MnO $\mathrm{Mns}_{2}$ NPs was only 39.7\%. When the GSH was added into the releasing medium, the release speed was increased obviously, and the release percentage of PLGA/AuNR/DTX@ $\mathrm{MnO}_{2}$ was increased to 78.1\%. Moreover, PLGA/AuNR/DTX@ $\mathrm{MnO}_{2}$ NPs have a pH-sensitive controlled release property. When the $\mathrm{pH}$ value decreased to 5.0, the release rates were increased, with or without GSH. These results demonstrated that the $\mathrm{MnO}_{2}$ nanosheets could prevent the drug release. Moreover, GSH and lower $\mathrm{pH}$ values can induce the drug release because of the $\mathrm{MnO}_{2}$ surface degradation. GSH level was higher and $\mathrm{pH}$ level was lower in the tumor region than in the normal tissues. ${ }^{21}$ Therefore, GSH/pH dual-responsive controlled release has great potential for this drug delivery system.
A

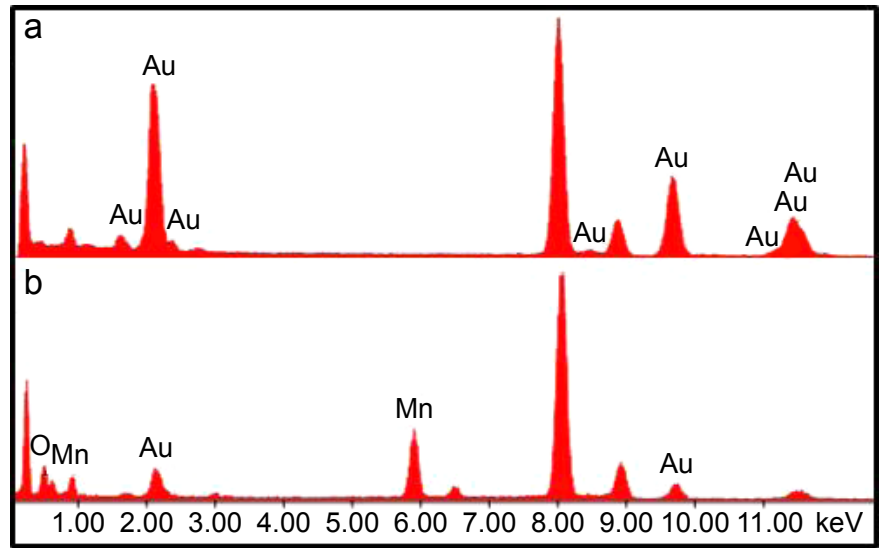

B

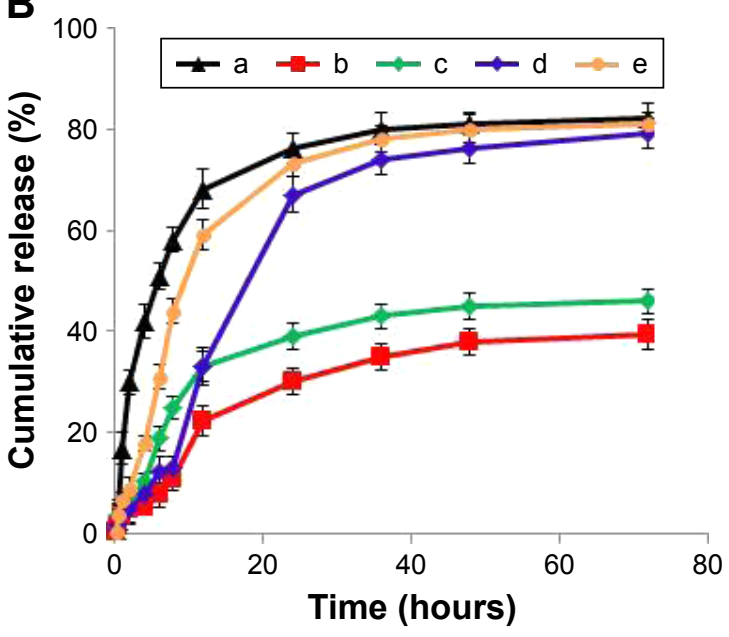

Figure 4 Characterization and determination of the drug release of PLGA/AuNRs/DTX@MnO .

Notes: (A) EDX analysis: (a) PLGA/AuNR/DTX and (b) PLGA/AuNR/DTX@MnO; ; (B) The cumulative release profile in vitro: (a) PLGA/AuNR/DTX, (b) PLGA/AuNR/ DTX@MnO +0 mM GSH (pH =7.4), (c) PLGA/AuNR/DTX@MnO +0 mM GSH (pH =5.0), (d) PLGA/AuNR/DTX@MnO +2 mM GSH (pH =7.4); and (e) PLGA/AuNR/ DTX@MnO +0 mM GSH $(\mathrm{pH}=5.0)$. The values are expressed as mean \pm standard deviation $(\mathrm{n}=3)$.

Abbreviations: AuNR, gold nanorod; DTX, docetaxel; EDX, energy-dispersive X-ray; GSH, glutathione; $\mathrm{MnO}_{2}$, manganese dioxide; PLGA, poly(lactic-co-glycolic acid). 


\section{In vitro $M R I$ and $X$-ray computed tomography (CT) imaging}

The previous studies reported that $\mathrm{MnO}_{2}$ can release $\mathrm{Mn}^{2+}$ ions in the tumor microenvironment. ${ }^{41}$ Therefore, $\mathrm{MnO}_{2}$ nanomaterials can be used for MRI in vitro and in vivo. ${ }^{22,42}$ In addition, the previous studies also showed that the gold nanomaterials can be used as the X-ray CT imaging contrast agents. ${ }^{29,38}$ In this drug delivery nanoplatform, AuNRs and $\mathrm{MnO}_{2}$ provided the dual-mode imaging for precise diagnosis of tumor. $T_{1}$-weighted MRI (Figure 5A) of PLGA/AuNR/ DTX@ $\mathrm{MnO}_{2} \mathrm{NP}$ solutions revealed that the MRI contrast was dependent on the concentration of delivery system and the GSH level. When the concentration of the solution increased, the brightness of $T_{1}$-weighted MR image was enhanced. The groups with GSH were brighter than the groups without GSH at the same concentration, revealing the capability of PLGA/AuNR/DTX@ $\mathrm{MnO}_{2}$ to be used as an effective $T_{1}$-weighted MRI contrast agent.

Figure 5C shows that the intensity of CT signal continuously increased with an increase in the concentration. The Hounsfield units were detected, indicating that PLGA/AuNR/ DTX@ $\mathrm{MnO}_{2}$ had great potential to be used as a positive $\mathrm{X}$-ray CT imaging contrast agent. The MRI and X-ray CT imaging results demonstrated that the PLGA/AuNR/DTX@ $\mathrm{MnO}_{2}$ NPs can be used for dual-mode imaging application for accurate tumor diagnosis.

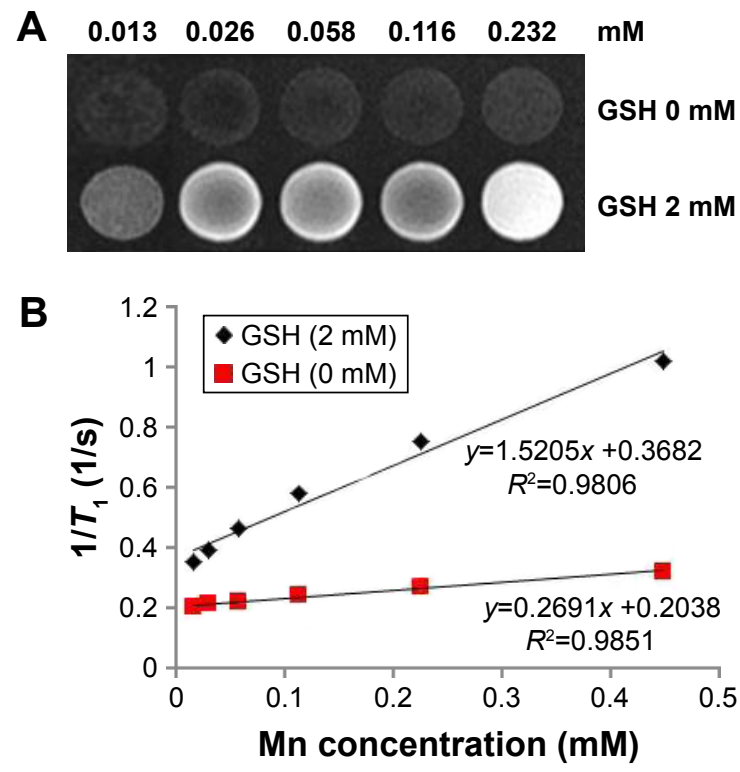

\section{Cellular uptake}

Whether the NPs enter into the cells is an important consideration for cancer therapy. Therefore, the cellular uptake of PLGA/AuNR/DTX and PLGA/AuNR/DTX@ $\mathrm{MnO}_{2}$ nanoplatforms was determined. The NPs were labeled with FITC fluorescence dye, and were tracked for the colocalization of FITC signal (green), and the cell nuclei were stained with DAPI (blue).

The fluorescence images showed that the NPs can enter into cells within $5 \mathrm{~h}$ (Figure 6A). The cellular uptake ratios of FITC, PLGA/AuNR/DTX, and PLGA/AuNR/DTX@ $\mathrm{MnO}_{2} \mathrm{NPs}$ were $7.61 \pm 0.38,99.7 \pm 0.14$, and $98.5 \% \pm 0.53 \%$, respectively (Figure 6B). It was found that there was no significant difference in the cellular uptake of NPs $(P>0.05)$, indicating that both of them can enter into the cells efficiently within $5 \mathrm{~h}$.

\section{Cell viability}

The combination of hyperthermia and chemotherapy is the efficient method for cancer treatment in clinical and laboratory research. Many anti-cancer drugs can be combined with hyperthermia treatment. The blood supply in tumor peripheral regions is generally hypervascular, and can be delivered easily. However, in the central area of the tumor, the scanty blood inflow causes difficulty in drug delivery, cell hypoxia, and poor heat dissipation (heat is
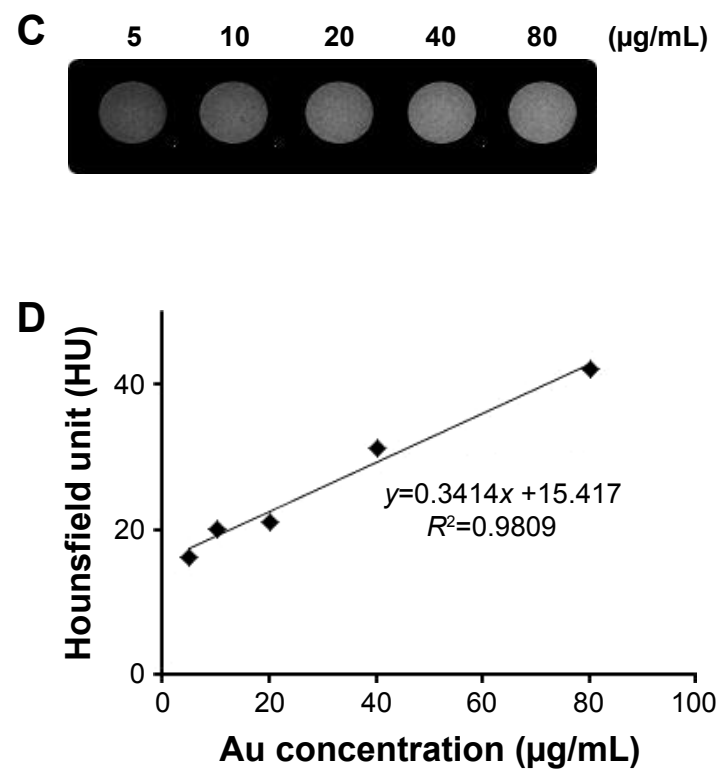

Figure 5 Dual-mode imaging in vitro.

Notes: (A) $T_{1}$-weighted MR images of PLGA/AuNR/DTX@MnO solutions at different $M n$ concentrations; (B) $T_{1}$ relaxation rate $R_{1}\left(I / T_{1}\right)$ against $M n$ concentration; (C) In vitro X-ray CT images of PLGA/AuNR/DTX@MnO suspended in PBS. The Au concentration ( $\mu \mathrm{g} / \mathrm{mL})$ in each sample is provided at the top of the respective images; (D) CT attenuation (HU) plot of PLGA/AuNR/DTX@MnO at various concentrations.

Abbreviations: AuNR, gold nanorod; CT, computed tomography; DTX, docetaxel; GSH, glutathione; MnO ${ }_{2}$, manganese dioxide; MR, magnetic resonance; PBS, phosphatebuffered saline; PLGA, poly(lactic-co-glycolic acid). 
A

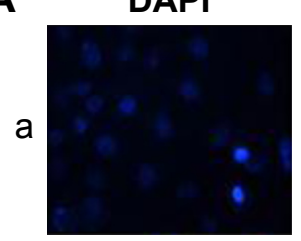

b
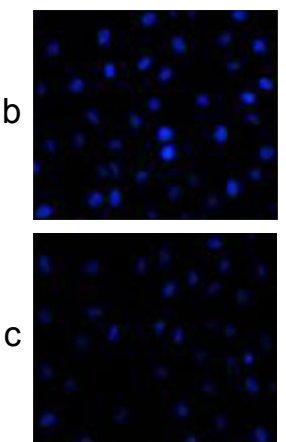

FITC
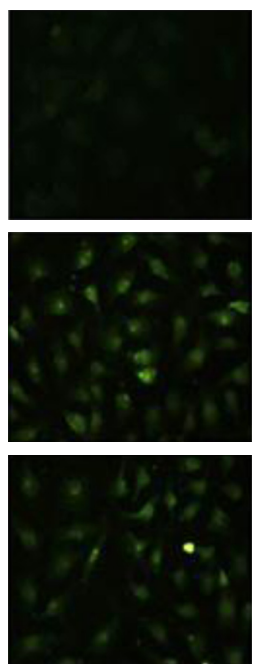

Merged
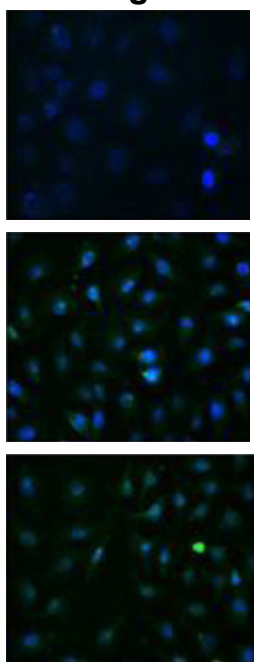

B

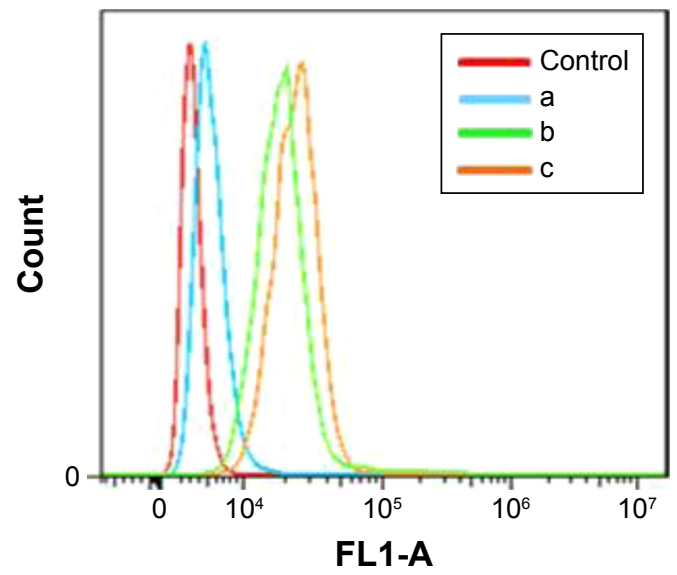

Figure 6 Cellular uptake.

Notes: (A) The fluorescence images of MCF-7 cells at 5 h: (a) FITC group, (b) PLGA/AuNR/DTX group, and (c) PLGA/AuNR/DTX@MnO group. (B) cellular uptake results of (a) FITC, (b) PLGA/AuNR/DTX, and (c) PLGA/AuNR/DTX@MnO at 5 h.

Abbreviations: AuNR, gold nanorod; DAPI, 4',6-diamidino-2-phenylindole; DTX, docetaxel; FITC, fluorescein isothiocyanate; MnO , manganese dioxide; PLGA, poly(lacticco-glycolic acid).

accumulated easily). Moreover, hypoxic cells are sensitive to hyperthermia. Therefore, hyperthermia and chemotherapy can complement each other and can be combined to enhance the anti-tumor effect.

In order to investigate the tumor growth inhibition in vitro, the cell viability of the NPs with or without RF treatment were investigated. As shown in Figure 7A, the PLGA/ AuNR@ $\mathrm{MnO}_{2}$ showed no significant toxicity to the cells, and there was no significant difference among DTX, PLGA/ DTX@ $\mathrm{MnO}_{2}$, and PLGA/AuNR/DTX@ $\mathrm{MnO}_{2}$ groups at various concentrations $(P>0.05)$. Figure $7 \mathrm{~B}$ shows that, after RF treatment, the cell viability of each group decreased. However, there was no significant difference between the DTX (or PLGA/DTX@ $\mathrm{MnO}_{2}$ ) group with and without RF irradiation at all the concentrations, $(P>0.05)$. Moreover, the cell viabilities of the groups which contained AuNRs showed a significant difference $(P<0.05)$ between these groups with and without RF treatment, demonstrating that the AuNRs could obviously produce heat when they were irradiated with RF.PLGA/AuNR/DTX@ $\mathrm{MnO}_{2}$ drug delivery system could
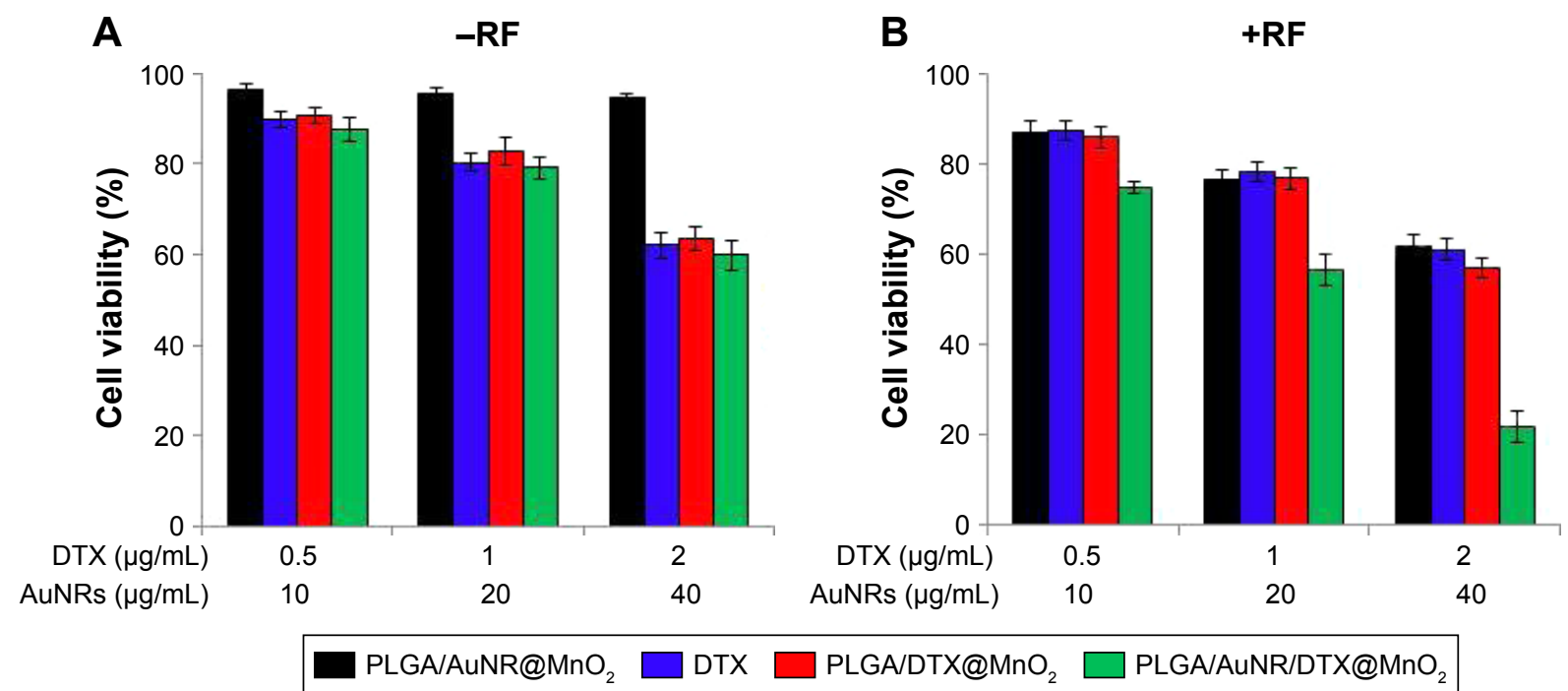

Figure 7 Cell viability of the NPs with or without RF treatment (I $3.56 \mathrm{MHz}, 400 \mathrm{~W}, 5 \mathrm{~min})$.

Notes: (A) Cell viability of the NPs without RF treatment. (B) Cell viability of the NPs with RF treatment. The values are expressed as mean \pm standard deviation ( $\mathrm{n}=3$ ). Abbreviations: AuNR, gold nanorod; DTX, docetaxel; $\mathrm{MnO}_{2}$, manganese dioxide; NPs, nanoparticles; PLGA, poly(lactic-co-glycolic acid); RF, radiofrequency. 
be used for the combined, AuNRs-induced, RF-responsive hyperthermia and chemotherapy.

\section{Cellular apoptosis}

The Annexin-V/PI kit was used to substantiate the enhanced apoptosis induced by the $\mathrm{MnO}_{2}$-based formulations. The FCM was performed three times, and the results (Figure 8) showed that the apoptosis rates of DTX, PLGA/ DTX/MnO 2 , PLGA/DTX/MnO + RF, PLGA/AuNR/DTX/ $\mathrm{MnO}_{2}$ and PLGA/AuNR/DTX/MnO $2+\mathrm{RF}$ groups were $23.3 \% \pm 1.24 \%, 24.3 \% \pm 1.23 \%, 25.1 \% \pm 1.72 \%, 27.2 \% \pm 1.95 \%$, and $43.8 \% \pm 2.32 \%$, respectively. Each of the DTX groups, namely the PLGA/DTX/MnO 2 and the PLGA/DTX/MnO ${ }_{2}+$ $\mathrm{RF}$ groups, showed no significant difference compared with the others $(P>0.05)$, indicating that PLGA/DTX/MnO ${ }_{2} \mathrm{NPs}$ were not sensitive to the RF irradiation treatment. After $\mathrm{RF}$ irradiation, the PLGA/AuNR/DTX/MnO $+\mathrm{RF}$ group showed higher apoptosis rate than the other groups $(P<0.05)$. In addition to the DTX effect, AuNRs-induced hyperthermia played a key role, demonstrating that the PLGA/AuNR/
DTX/ $\mathrm{MnO}_{2}$ drug delivery system could induce cellular apoptosis effectively.

\section{Anti-tumor effect in vivo}

For the investigation of the anti-tumor effect, the relative tumor volume and the body weights of the mice were detected every other day. Figure 9A showed that the PLGA/ AuNR/DTX@ $\mathrm{MnO}_{2}+\mathrm{RF}$ had obvious anti-tumor effect and had significant difference compared with the other groups $(P<0.05)$. Moreover, compared with the relative tumor volume of PLGA/DTX@MnO ${ }_{2}$, the PLGA/DTX@ $\mathrm{MnO}_{2}+\mathrm{RF}$ group showed no significant difference, indicating that the AuNRs played the key role for RF-responsive hyperthermia. As in the DTX group, no weight loss was found in the other groups, suggesting that the toxicity of all the drug delivery systems was negligible (Figure 9B).

For further detection of the anti-tumor effect, the cell morphology of the tumor tissues was investigated by using H\&E staining method (Figure 9C). The H\&E sections of PLGA/AuNR/DTX@MnO + RF group showed high cellular
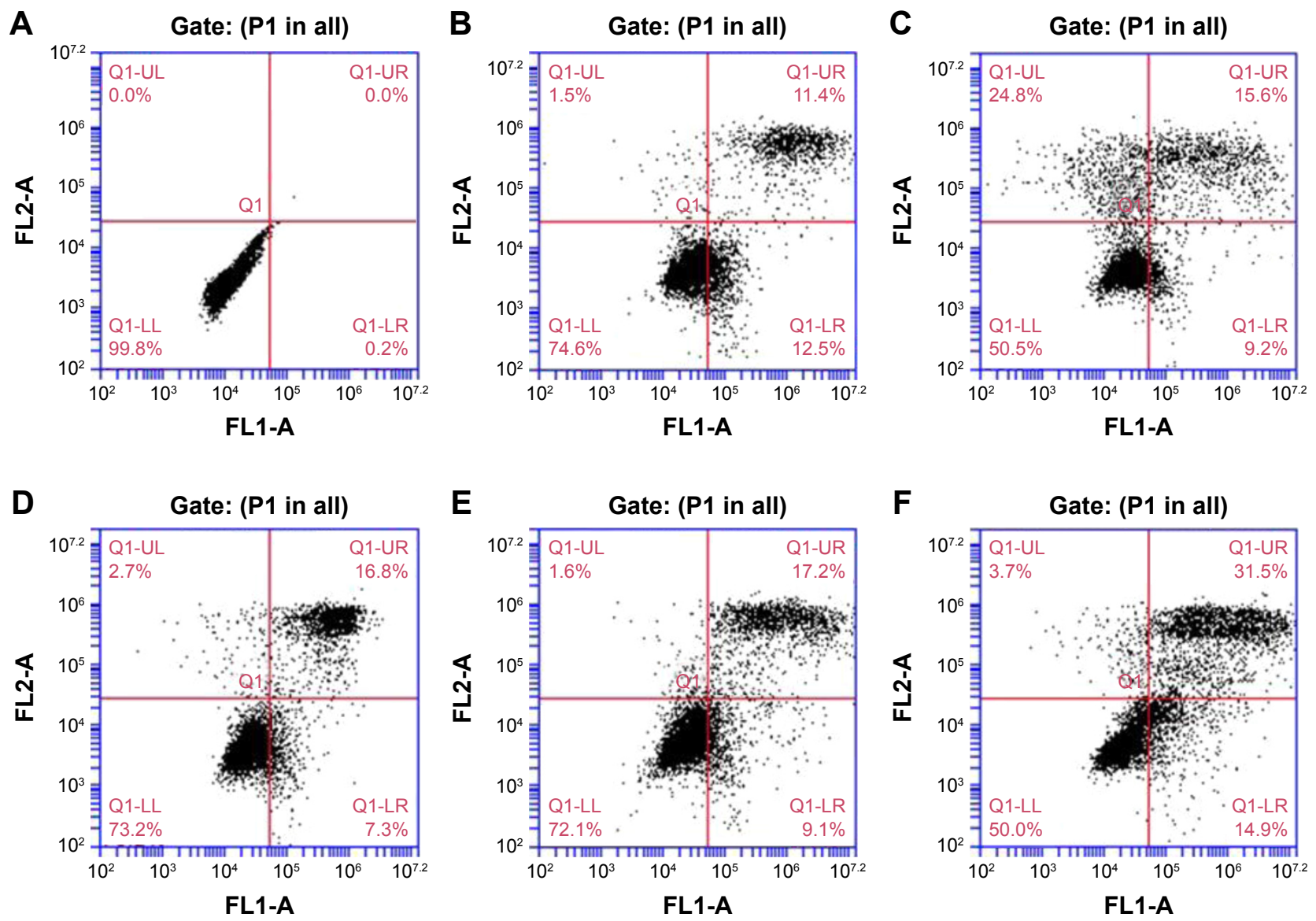

Figure 8 Cell apoptosis of the experimental groups.

Notes: (A) Control; (B) DTX; (C) PLGA/DTX/MnO ; (D) PLGA/DTX/MnO + RF; (E) PLGA/AuNR/DTX/MnO ; (F) PLGA/AuNR/DTX/MnO + RF.

Abbreviations: AuNR, gold nanorod; DTX, docetaxel; $\mathrm{MnO}_{2}$, manganese dioxide; PLGA, poly(lactic-co-glycolic acid); RF, radiofrequency. 
A

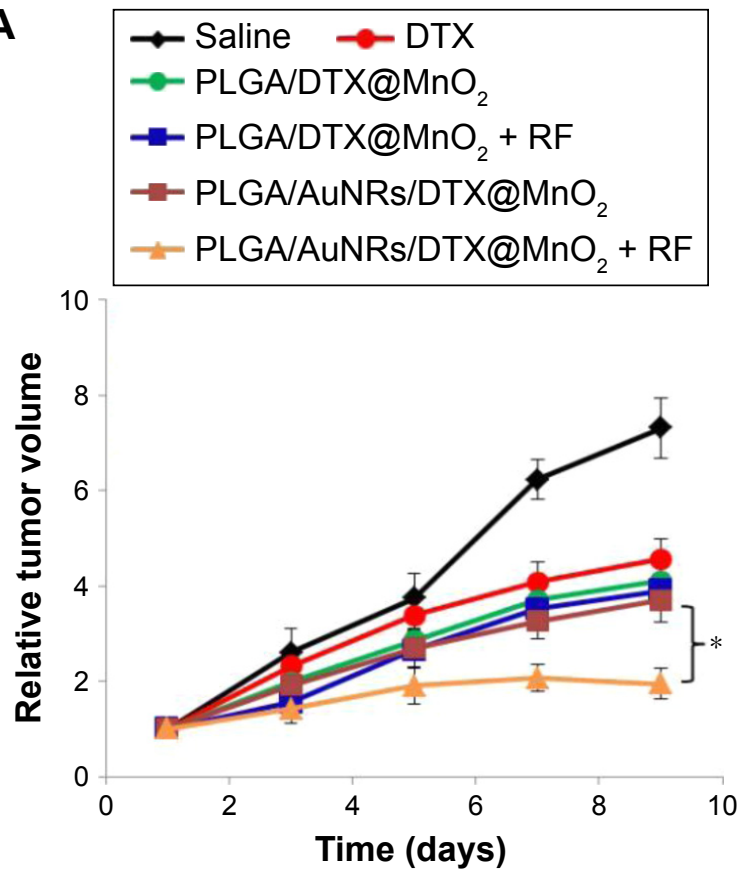

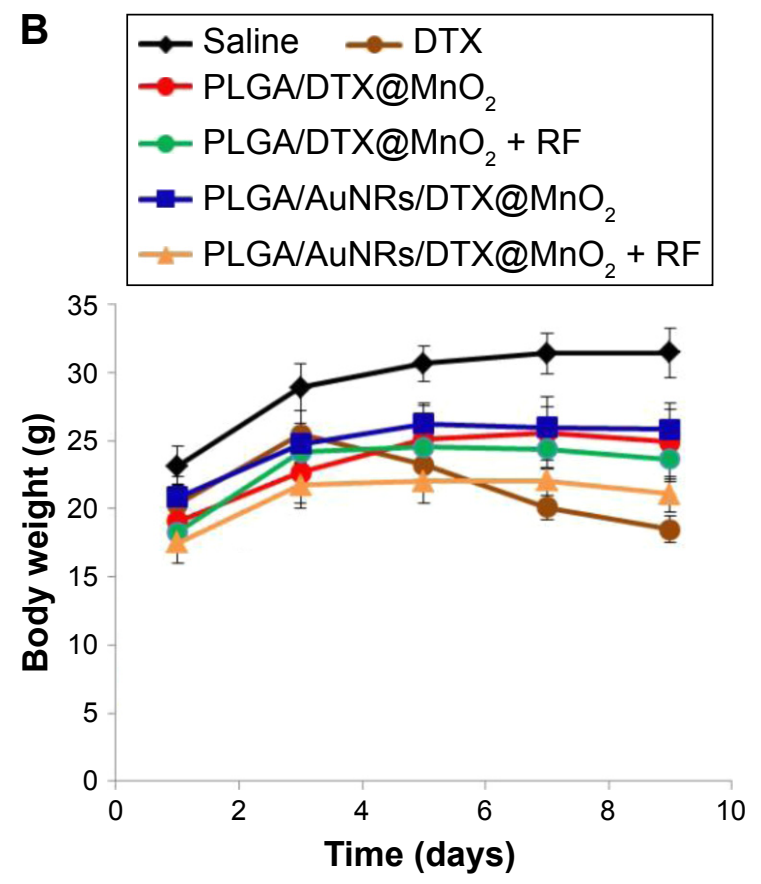

C
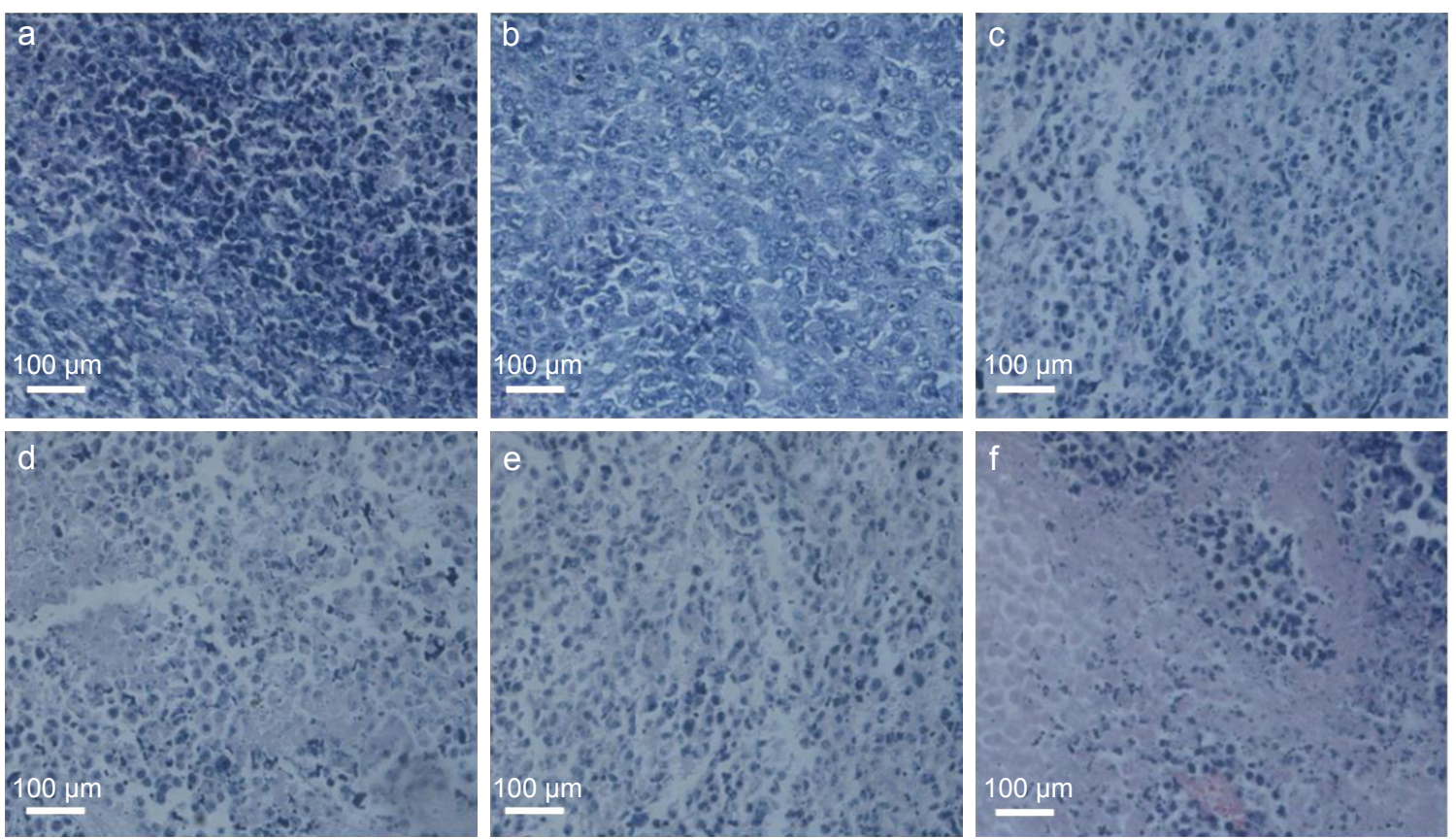

Figure 9 Inhibition of tumor growth in vivo by the drug delivery system.

Notes: (A) Relative volumes of tumor-bearing mice; (B) body weights of the mice models (mean \pm standard deviation; $n=6$ ); (C) H\&E-stained tumor tissue sections: (a) control, (b) DTX, (c) PLGA/DTX/MnO, (d) PLGA/DTX/MnO + RF, (e) PLGA/AuNR/DTX/MnO, and (f) PLGA/AuNR/DTX/MnO + RF.

Abbreviations: H\&E, hematoxylin and eosin; AuNR, gold nanorod; DTX, docetaxel; $\mathrm{MnO}_{2}$, manganese dioxide; PLGA, poly(lactic-co-glycolic acid); RF, radiofrequency.

polymorphism, cell necrosis, cell lysis, and cell fragment. The cell morphologies of the DTX, PLGA/DTX@ $\mathrm{MnO}_{2}$, PLGA/DTX@MnO + RF, and PLGA/AuNR/DTX@ $\mathrm{MnO}_{2}$ groups were similar, and the level of cell deformation was less than that in the PLGA/AuNR/DTX@ $\mathrm{MnO}_{2}+\mathrm{RF}$ group. The results indicated that the combination therapy of AuNRsinduced RF hyperthermia and DTX-induced chemotherapy could be used to achieve efficient tumor inhibition.

\section{Biodistribution}

In order to investigate the biodistribution of DTX alone or PLGA/AuNR/DTX@ $\mathrm{MnO}_{2}$ drug delivery system, the HPLC method was used in this study. As shown in Figure 10, it was observed that a significant difference existed between the DTX and PLGA/AuNR/DTX@ $\mathrm{MnO}_{2}$ groups. DTX-alone group was mainly distributed in liver, lung, and kidney, where PLGA/AuNR/DTX@MnO $\mathrm{Mn}_{2}$ was mainly distributed in lung 

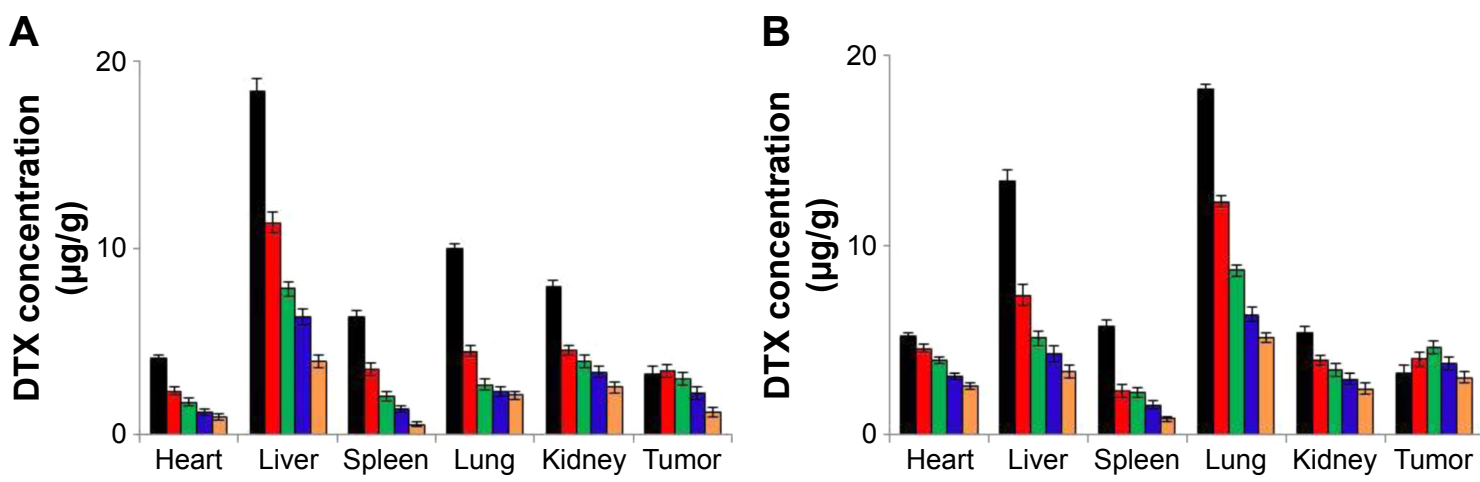

$\square 1 \mathrm{~h} \square 2 \mathrm{~h} \square 4 \mathrm{~h} \square 6 \mathrm{~h} \square 8 \mathrm{~h}$

Figure 10 Biodistribution of the drug delivery system at I, 2, 4, and $8 \mathrm{~h}$, respectively.

Notes: (A) DTX; (B) PLGA/AuNR/DTX/MnO . The values are expressed as mean \pm standard deviation $(n=6)$.

Abbreviations: AuNR, gold nanorod; DTX, docetaxel; $\mathrm{MnO}_{2}$, manganese dioxide; PLGA, poly(lactic-co-glycolic acid).

and liver organs. Importantly, the uptake of DTX in tumor was significantly higher in the PLGA/AuNR/DTX@ $\mathrm{MnO}_{2}$ group than in the DTX group at 4,6 , and $8 \mathrm{~h}$, respectively $(P<0.05)$. In addition, the biodistribution of PLGA/AuNR/ DTX@ $\mathrm{MnO}_{2}$ was the highest at $4 \mathrm{~h}$ after administration.

\section{Effects of MRI and X-ray imaging in vivo}

The diagnostic imaging of the tumor is very important for prognosis, and it is good for the real-time tracking of the therapeutic effect of the drug delivery system. Nowadays, a number of theranostic drug delivery systems have been prepared and investigated in vitro and in vivo. ${ }^{43-45}$ In the previous studies, it was found that $\mathrm{Mn}^{2+}$ and gold nanomaterials can be used for MRI and X-ray CT imaging for the early diagnosis of cancer, respectively. ${ }^{41,46}$ Gold nanoshells and $\mathrm{Fe}_{3} \mathrm{O}_{4}$ NPs have been used to prepare a core-shell structured delivery system that had the dual-mode imaging effect. ${ }^{33}$ Kim et al ${ }^{47}$ used $\mathrm{Mn}^{2+}$ and $\mathrm{Fe}_{3} \mathrm{O}_{4}$ to prepare a delivery system, showing the $T_{1} / T_{2}$ dual-mode MRI effect. Nowadays, in cancer research, a number of dual-mode imaging drug delivery systems have been constructed for tumor theranostic applications ${ }^{48,49}$ as they have more advantages. On the one hand, the dual-mode imaging can be more accurate to determine the real-time location of the drug delivery system. For better use of RF hyperthermia, the time point of treatment is very important. It is possible to determine the maximum distribution of the drug delivery system at the tumor site by using this imaging method. On the other hand this method can also detect the best time point for hyperthermia.

MRI and CT are the most commonly used and efficient imaging methods in clinical treatments. In this study, dualmode imaging (MRI and X-ray CT imaging) was used for tumor diagnostics (Figure 11). PLGA/AuNR/DTX@, $\mathrm{MnO}_{2}$ solution was injected through the mouse tail vein, and the results were obtained at 4 and $8 \mathrm{~h}$ after injection. It can be seen that the administration of PLGA/AuNR/DTX@ $\mathrm{MnO}_{2}$ enabled a clear MR or CT contrast imaging at the tumor site, and both the MRI and CT results showed clearly that the
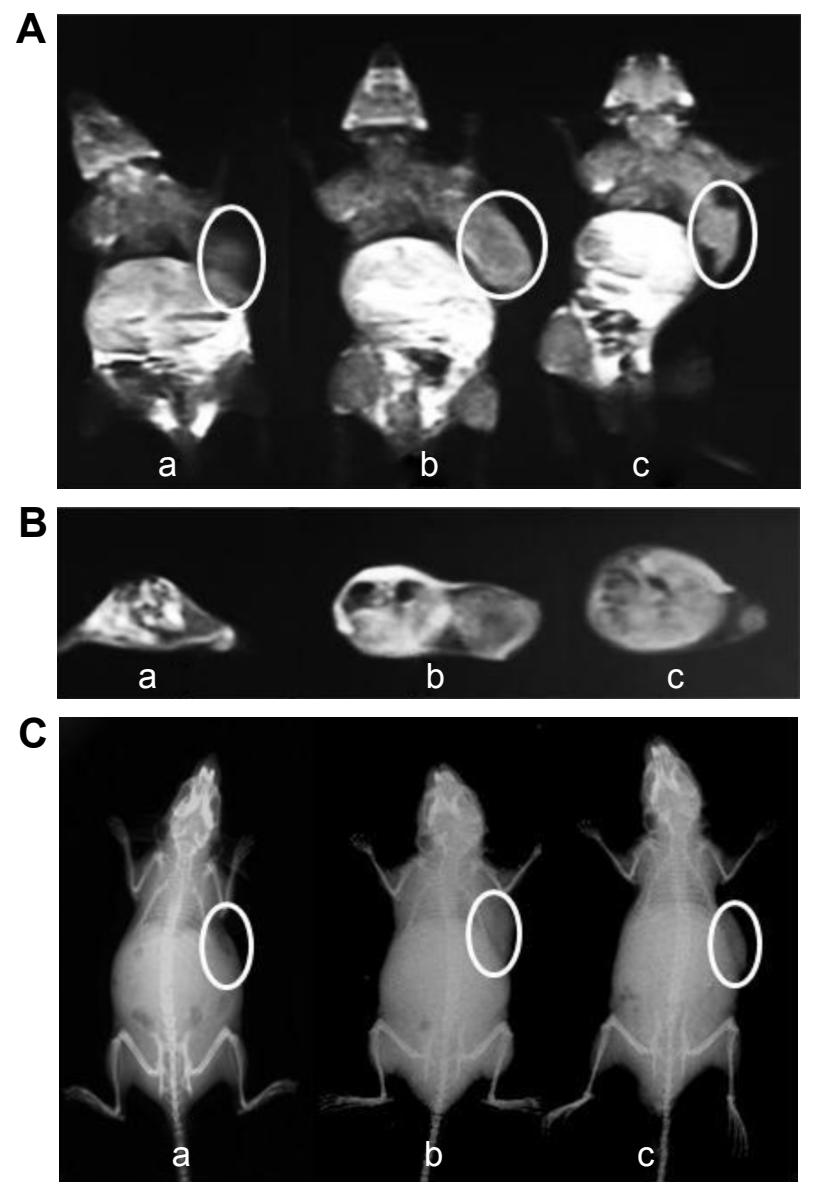

Figure I I Dual-mode imaging in vivo.

Notes: (A, B) $T_{1}$-weighted MR images; (C) X-ray CT images: (a) control; (b) $4 \mathrm{~h}$; (c) $8 \mathrm{~h}$.

Abbreviations: MR, magnetic resonance; CT, computed tomography. 
biodistribution of PLGA/AuNR/DTX@ $\mathrm{MnO}_{2}$ in the tumor site was the highest at $4 \mathrm{~h}$ after administration (Figure S2). It was shown that this drug delivery system had great potential in tumor dual-mode imaging applications.

\section{Conclusion}

In this study, the PLGA/AuNR/DTX@MnO ${ }_{2}$ drug delivery system was constructed and investigated in vitro and in vivo. The AuNRs with different ARs were prepared first, and it was proven that they could be used for RF-induced hyperthermia. All the results indicated that the PLGA/AuNR/DTX@ $\mathrm{MnO}_{2}$ drug delivery system could be used for chemotherapy, hyperthermia, controlled release, and dual-mode imaging. The PLGA/AuNR/DTX@ $\mathrm{MnO}_{2}$ delivery system has great potential to be used in tumor theranostic applications.

\section{Acknowledgments}

This work was supported by the National Natural Science Foundation of China (Nos 81673021, 81573151, 81572991, and 81302717), the China Postdoctoral Science Foundation (Nos 2014M562002 and 2015T80783), and Outstanding Young Talent Research Fund of Zhengzhou University (No 1421331073).

\section{Disclosure}

The authors report no conflicts of interest in this work.

\section{References}

1. Myrehaug S, Sahgal A, Russo SM, et al. Stereotactic body radiotherapy for pancreatic cancer: recent progress and future directions. Expert Rev Anticancer Ther. 2016;16:523-530.

2. Radecka B, Litwiniuk M. Breast cancer in young women. Ginekol Pol. 2016;87:659-663.

3. Cheng T, Liu J, Ren J, et al. Green tea catechin-based complex micelles combined with doxorubicin to overcome cardiotoxicity and multidrug resistance. Theranostics. 2016;6:1277-1292.

4. Chen MC, Lin ZW, Ling MH. Near-infrared light-activatable microneedle system for treating superficial tumors by combination of chemotherapy and photothermal therapy. ACS Nano. 2016;10:93-101.

5. Su S, Ding Y, Li Y, Wu Y, Nie G. Integration of photothermal therapy and synergistic chemotherapy by a porphyrin self-assembled micelle confers chemosensitivity in triple-negative breast cancer. Biomaterials. 2016;80:169-178.

6. Luo L, Bian Y, Liu Y, et al. Gold nanoshells: combined near infrared photothermal therapy and chemotherapy using gold nanoshells coated liposomes to enhance antitumor effect. Small. 2016;12:4102.

7. Xing R, Liu K, Jiao T, et al. An injectable self-assembling collagen-gold hybrid hydrogel for combinatorial antitumor photothermal/photodynamic therapy. Adv Mater. 2016;28:3669-3676.

8. Sugiura T, Matsuki D, Okajima J, et al. Photothermal therapy of tumors in lymph nodes using gold nanorods and near-infrared laser light with controlled surface cooling. Nano Res. 2015;8:3842-3852.

9. Lin CC, Cheng YT, Chen MW, Lin SM. The effectiveness of multiple electrode radiofrequency ablation in patients with hepatocellular carcinoma with lesions more than $3 \mathrm{~cm}$ in size and barcelona clinic liver cancer stage A to B2. Liver Cancer. 2016;5:8-20.
10. He ZX, Xiang P, Gong JP, Cheng NS, Zhang W. Radiofrequency ablation versus resection for Barcelona clinic liver cancer very early/early stage hepatocellular carcinoma: a systematic review. Ther Clin Risk Manag. 2016;12:295-303.

11. Wang W, Despanie J, Shi P, et al. Lacritin-mediated regeneration of the corneal epithelia by protein polymer nanoparticles. J Mater Chem B Mater Biol Med. 2014;2:8131-8141.

12. Song J, Yang X, Jacobson O, et al. Sequential drug release and enhanced photothermal and photoacoustic effect of hybrid reduced graphene oxide-loaded ultrasmall gold nanorod vesicles for cancer therapy. ACS Nano. 2015;9:9199-9209.

13. Wang XW, Gao W, Fan H, et al. Simultaneous tracking of drug molecules and carriers using aptamer-functionalized fluorescent superstable gold nanorod-carbon nanocapsules during thermo-chemotherapy. Nanoscale. 2016;8:7942-7948.

14. Collins CB, McCoy RS, Ackerson BJ, Collins GJ, Ackerson CJ. Radiofrequency heating pathways for gold nanoparticles. Nanoscale. 2014;6:8459-8472.

15. Rejinold NS, Jayakumar R, Kim YC. Radio frequency responsive nanobiomaterials for cancer therapy. J Control Release. 2015;204:85-97.

16. Golubev AA, Prilepskii AY, Dykman LA, Khlebtsov NG, Bogatyrev VA. Colorimetric evaluation of the viability of the microalga dunaliella salina as a test tool for nanomaterial toxicity. Toxicol Sci. 2016; 151:115-125.

17. Zhuang B, Du L, Xu HX, et al. Self-assembled micelle loading cabazitaxel for therapy of lung cancer. Int J Pharm. 2016;499:146-155.

18. Su ZH, Liang YC, Yao Y, Wang TQ, Zhang N. Polymeric complex micelles based on the double-hydrazone linkage and dual drug-loading strategy for $\mathrm{pH}$-sensitive docetaxel delivery. J Mat Chem B. 2016;4: 1122-1133.

19. Chakravarty S, Unold J, Shuboni-Mulligan DD, Blanco-Fernandez B, Shapiro EM. Surface engineering of bismuth nanocrystals to counter dissolution. Nanoscale. 2016;8:13217-13222.

20. Zhao Y, Ren W, Zhong T, et al. Tumor-specific pH-responsive peptidemodified $\mathrm{pH}$-sensitive liposomes containing doxorubicin for enhancing glioma targeting and anti-tumor activity. J Control Release. 2016; 222:56-66.

21. Gao C, Liu T, Dang Y, et al. $\mathrm{pH} /$ redox responsive core cross-linked nanoparticles from thiolated carboxymethyl chitosan for in vitro release study of methotrexate. Carbohydr Polym. 2014;111:964-970.

22. Hao Y, Wang L, Zhang B, et al. Multifunctional nanosheets based on folic acid modified manganese oxide for tumor-targeting theranostic application. Nanotechnology. 2015;27:025101.

23. Han X, Chen J, Jiang M, et al. Paclitaxel-paclitaxel prodrug nanoassembly as a versatile nanoplatform for combinational cancer therapy. ACS Appl Mater Interfaces. 2016;8:33506-33513.

24. Hou L, Yang X, Ren J, et al. A novel redox-sensitive system based on single-walled carbon nanotubes for chemo-photothermal therapy and magnetic resonance imaging. Int J Nanomedicine. 2016;11:607-624.

25. Kim H, Kim J, Lee M, Choi HC, Kim WJ. Stimuli-regulated enzymatically degradable smart graphene-oxide-polymer nanocarrier facilitating photothermal gene delivery. Adv Healthc Mater. 2016;5: 1918-1930.

26. Xiong H, Guo Z, Zhang W, Zhong H, Liu S, Ji Y. Redox-responsive biodegradable PEGylated nanographene oxide for efficiently chemophotothermal therapy: a comparative study with non-biodegradable PEGylated nanographene oxide. J Photochem Photobiol B. 2014;138: 191-201.

27. Ren F, Bhana S, Norman DD, et al. Gold nanorods carrying paclitaxel for photothermal-chemotherapy of cancer. Bioconjugate Chem. 2013; 24:376-386.

28. Nguyen SC, Zhang Q, Manthiram K, et al. Study of heat transfer dynamics from gold nanorods to the environment via time-resolved infrared spectroscopy. ACS Nano. 2016;10:2144-2151.

29. Hao Y, Zhang B, Zheng C, et al. The tumor-targeting core-shell structured DTX-loadedPLGA@Au nanoparticles for chemo-photothermal therapy and X-ray imaging. J Control Rel. 2015;220:545-555. 
30. Qiu Y, Palankar R, Echeverria M, Medvedev N, Moya SE, Delcea M. Design of hybrid multimodal poly(lactic-co-glycolic acid) polymer nanoparticles for neutrophil labeling, imaging and tracking. Nanoscale. 2013;5:12624-12632.

31. Yang X, He D, He X, et al. Glutathione-mediated degradation of surfacecapped $\mathrm{MnO}_{2}$ for drug release from mesoporous silica nanoparticles to cancer cells. Part Part Syst Char. 2015;32:205-212.

32. Hao Y, Wang L, Zhao Y, et al. Targeted imaging and chemo-phototherapy of brain cancer by a multifunctional drug delivery system. Macromol Biosci. 2015;15:1571-1585.

33. Wang L, Zhang P, Shi J, et al. Radiofrequency-triggered tumor-targeting delivery system for theranostics application. ACS Appl Mater Interfaces. 2015;7:5736-5747.

34. Burrows ND, Lin W, Hinman JG, et al. Surface chemistry of gold nanorods. Dioxide nanoparticles enhance chemotherapy response by priming Langmuir. 2016;32:9905-9921.

35. Vijayaraghavan P, Liu CH, Hwang KC. Synthesis of multibranched gold nanoechinus using a gemini cationic surfactant and its application for surface enhanced raman scattering. ACS Appl Mater Interfaces. 2016;8:23909-23919.

36. Su G, Yang C, Zhu JJ. Fabrication of gold nanorods with tunable longitudinal surface plasmon resonance peaks by reductive dopamine. Langmuir. 2015;31:817-823.

37. Wang X, Li G, Ding Y, Sun S. Understanding the photothermal effect of gold nanostars and nanorods for biomedical applications. RSC Adv. 2014;4:30375-30383.

38. Wang L, Meng D, Hao Y, et al. A gold nanostar based multi-functional tumor-targeting nanoplatform for tumor theranostic applications. J Mat Chem B. 2016;4:5895-5906.

39. Yang X, He DG, He XX, et al. Glutathione-mediated degradation of surface-capped $\mathrm{MnO}_{2}$ for drug release from mesoporous silica nanoparticles to cancer cells. Part Part Syst Char. 2015;32:205-212.
40. Joshi N, Rawat K, Bohidar HB. Influence of structure, charge, and concentration on the pectin-calcium-surfactant complexes. JPhys Chem B. 2016;120:4249-4257.

41. Hao Y, Wang L, Zhang B, et al. Multifunctional nanosheets based on folic acid modified manganese oxide for tumor-targeting theranostic application. Nanotechnology. 2016;27:025101.

42. Song M, Liu T, Shi C, Zhang X, Chen X. Bioconjugated manganese dioxide nanoparticles enhance chemotherapy response by priming tumorassociated macrophages toward M1-like phenotype and attenuating tumor hypoxia. ACS Nano. 2016;10:633-647.

43. Lee MY, Lee C, Jung HS, et al. Biodegradable photonic melanoidin for theranostic applications. ACS Nano. 2016;10:822-831.

44. Lin TY, Li Y, Liu Q, et al. Novel theranostic nanoporphyrins for photodynamic diagnosis and trimodal therapy for bladder cancer. Biomaterials. 2016;104:339-351.

45. Li W, Zheng C, Pan Z, et al. Smart hyaluronidase-actived theranostic micelles for dual-modal imaging guided photodynamic therapy. Biomaterials. 2016;101:10-19.

46. Wang L, Meng D, Hao Y, et al. Gold nanostars mediated combined photothermal and photodynamic therapy and X-ray imaging for cancer theranostic applications. J Biomater Appl. 2015;30:547-557.

47. Kim MH, Son HY, Kim GY, Park K, Huh YM, Haam S. Redoxable heteronanocrystals functioning magnetic relaxation switch for activatable T1 and T2 dual-mode magnetic resonance imaging. Biomaterials. 2016;101:121-130.

48. Ni D, Shen Z, Zhang J, et al. Integrating anatomic and functional dual-mode magnetic resonance imaging: design and applicability of a bifunctional contrast agent. ACS Nano. 2016;10:3783-3790.

49. Yang H, Li T, Chen Y, Xu M, Li Y. VCAM-1-targeted silica nanoparticles doped with FITC and $\mathrm{Fe}_{3} \mathrm{O}_{4}$ for dual-mode cellular imaging of LPS-induced inflammatory endothelial cells. J Control Rel. 2015 213:e40-e41. 


\section{Supplementary materials}

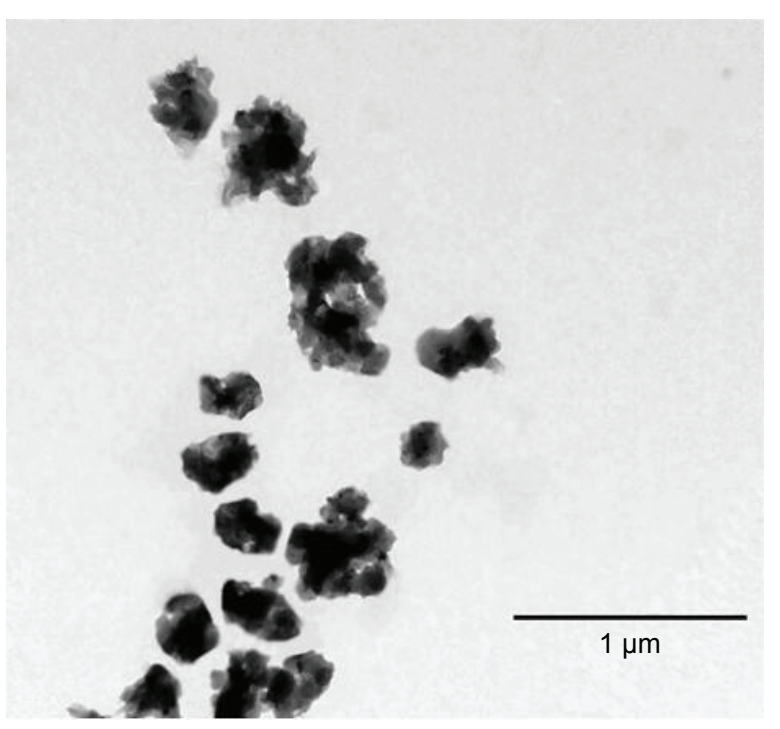

Figure SI TEM image of PLGA/AuNR/DTX@MnO .

Abbreviations: TEM, transmission electron microscopy; AuNR, gold nanorod; DTX, docetaxel; $\mathrm{MnO}_{2}$, manganese dioxide; PLGA, poly(lactic-co-glycolic acid).

Table SI Particle sizes

\begin{tabular}{lll}
\hline Nano-suspension & PBS & Physiologically mimicked environment \\
\hline PLGA/DTX & $239.0 \pm 3.7 \mathrm{~nm}$ & $240.4 \pm 3.5 \mathrm{~nm}$ \\
PLGA/AuNR/DTX & $263.3 \pm 4.5 \mathrm{~nm}$ & $265.5 \pm 3.9 \mathrm{~nm}$ \\
PLGA/AuNR/DTX@MnO & $282.1 \pm 6.2 \mathrm{~nm}$ & $287.2 \pm 6.5 \mathrm{~nm}$ \\
\hline
\end{tabular}

Abbreviations: AuNR, gold nanorod; DTX, docetaxel; $\mathrm{MnO}_{2}$, manganese dioxide; PBS, phosphate-buffered saline; PLGA, poly(lactic-co-glycolic acid).

Table S2 Zeta potential

\begin{tabular}{lll}
\hline Nano-suspension & PBS & Physiologically mimicked environment \\
\hline PLGA/DTX & $-15.3 \pm 1.6 \mathrm{mV}$ & $-15.1 \pm 1.5 \mathrm{mV}$ \\
PLGA/AuNR/DTX & $-12.3 \pm 1.5 \mathrm{mV}$ & $-12.5 \pm 1.3 \mathrm{mV}$ \\
PLGA/AuNR/DTX@MnO & $-9.7 \pm 1.4 \mathrm{mV}$ & $-9.4 \pm 1.6 \mathrm{mV}$ \\
\hline
\end{tabular}

Abbreviations: AuNR, gold nanorod; DTX, docetaxel; $\mathrm{MnO}_{2}$, manganese dioxide; PLGA, poly(lactic-co-glycolic acid).
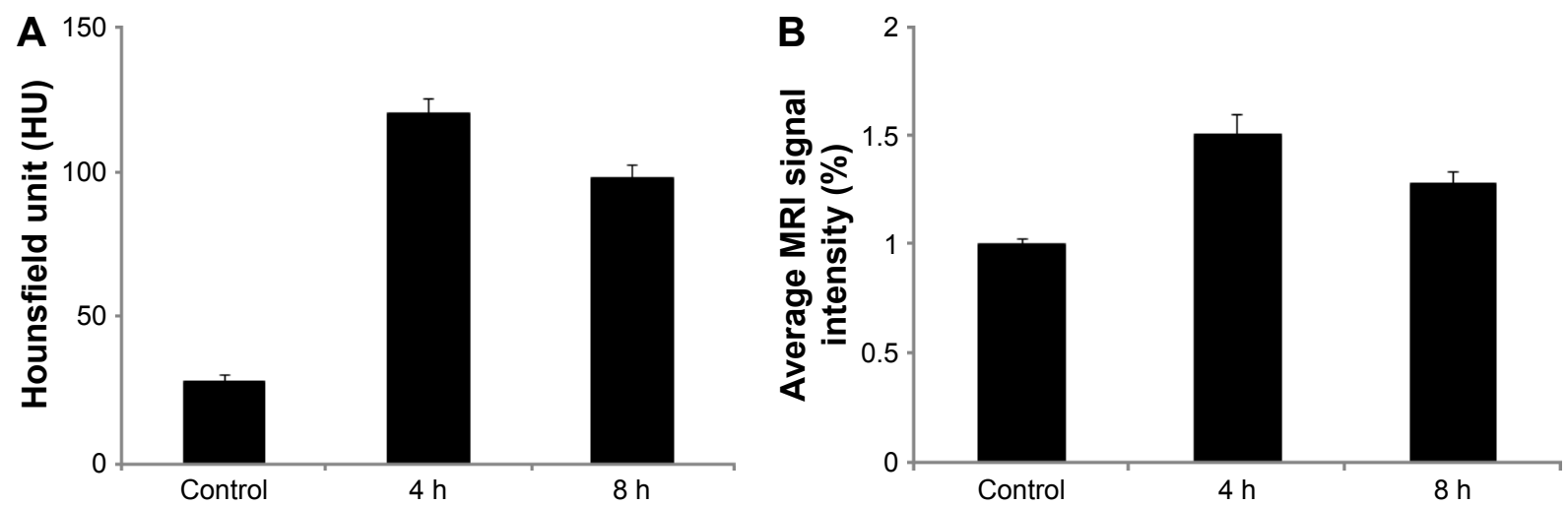

Figure S2 (A) HU value of the tumor at 4 and $8 \mathrm{~h}$ after the injection of PLGA/AuNR/DTX@MnO ; (B) the relative signal intensity of the tumor at 4 and $8 \mathrm{~h}$ after the injection of PLGA/AuNR/DTX@MnO .

Note: The values are expressed as mean \pm standard deviation $(n=3)$.

Abbreviations: AuNR, gold nanorod; DTX, docetaxel; $\mathrm{MnO}_{2}$, manganese dioxide; PLGA, poly(lactic-co-glycolic acid); HU, Hounsfield units; MRI, magnetic resonance imaging. 
International Journal of Nanomedicine

Dovepress

\section{Publish your work in this journal}

The International Journal of Nanomedicine is an international, peerreviewed journal focusing on the application of nanotechnology in diagnostics, therapeutics, and drug delivery systems throughou the biomedical field. This journal is indexed on PubMed Central, MedLine, CAS, SciSearch ${ }^{\circledR}$, Current Contents ${ }^{\circledR} /$ Clinical Medicine,
Journal Citation Reports/Science Edition, EMBase, Scopus and the Elsevier Bibliographic databases. The manuscript management system is completely online and includes a very quick and fair peer-review system, which is all easy to use. Visit http://www.dovepress.com/ testimonials.php to read real quotes from published authors.

Submit your manuscript here: http://www.dovepress.com/international-journal-of-nanomedicine-journal 